\title{
Dilaton in a soft-wall holographic approach to mesons and baryons
}

\author{
Thomas Gutsche ${ }^{1}$, Valery E. Lyubovitskij ${ }^{1}{ }^{*}$, Ivan Schmidt $^{2}$, Alfredo Vega ${ }^{2}$ \\ 1 Institut für Theoretische Physik, Universität Tübingen, \\ Kepler Center for Astro and Particle Physics, \\ Auf der Morgenstelle 14, D-72076 Tübingen, Germany \\ 2 Departamento de Física y Centro Científico Tecnológico de Valparaíso (CCTVal), \\ Universidad Técnica Federico Santa María, \\ Casilla 110-V, Valparaíso, Chile
}

(Dated: July 3, 2018)

\begin{abstract}
We discuss a holographic soft-wall model developed for the description of mesons and baryons with adjustable quantum numbers $n, J, L, S$. This approach is based on an action which describes hadrons with broken conformal invariance and which incorporates confinement through the presence of a background dilaton field. We show that in the case of the bound-state problem (hadronic mass spectrum) two versions of the model with a positive and negative dilaton profile are equivalent to each other by a special transformation of the bulk field. We also comment on recent works which discuss the dilaton sign in the context of soft-wall approaches.
\end{abstract}

PACS numbers: 11.10.Kk, 11.25.Tq, 14.20.-c, 14.40.-n

Keywords: soft-wall holographic model, hadrons, mass spectrum

\section{INTRODUCTION}

Based on the correspondence of string theory in anti-de Sitter (AdS) space and conformal field theory (CFT) in physical space-time [1], a class of AdS/QCD approaches was recently successfully developed for describing the phenomenology of hadronic properties. In order to break conformal invariance and incorporate confinement in the infrared (IR) region two alternative AdS/QCD backgrounds have been suggested in the literature: the "hard-wall" approach [2]-[4], based on the introduction of an IR brane cutoff in the fifth dimension, and the "soft-wall" approach [5]22], based on using a soft cutoff. This last procedure can be introduced in the following ways: i) as a background field (dilaton) in the overall exponential of the action ("dilaton" soft-wall model), ii) in the warping factor of the AdS metric ("metric" soft-wall model), iii) in the effective potential of the action. These methods could in principle be equivalent to each other due to a redefinition of the bulk field involving the dilaton or by a redefinition of the effective potential. Moreover, in Ref. [23] a so-called no-wall holographic model was recently proposed. This approach is motivated by the soft-wall model, containing the dilaton field in the exponential prefactor [5 9]. After a special dilaton transformation, the prefactor is moved to the effective potential, where the dilaton field is replaced by a dilaton condensate. We would like to stress that the equivalence between the different versions of the soft-wall models is important to guarantee that they describe the same physics. In particular, below we show how to match the "metric" soft-wall model to the "dilaton" soft-wall model that both are equivalent in describing the hadronic phenomenology. Application of the "metric" models to other problems, like e.g. the hierarchy problem in the Randall-Sundrum model [25] goes beyond the scope of the present discussion.

In the literature there exist detailed discussions of the sign of the dilaton profile in the dilaton exponential $\exp ( \pm \varphi(z))[5,9,21,22]$ for the soft-wall model (for a discussion of the sign of the dilaton in the warping factor of the AdS metric see Refs. [10]). The negative sign was suggested in Ref. [5] and recently discussed in Ref. 22]. It leads to a Regge-like behavior of the meson spectrum, including a straightforward extension to fields of higher spin $J$. Also, in Ref. [22] it was shown that this choice of the dilaton sign guarantees the absence of a spurious massless scalar mode in the vector channel of the soft-wall model. We stress that alternative versions of this model with positive sign are also possible, but they are restricted in applications. In particular, the version with a positive dilaton is obtained by redefinition of the bulk field $V(x, z)$ as $V(x, z)=\exp (\varphi) \tilde{V}(x, z)$, where the transformed field corresponds

* On leave of absence from Department of Physics, Tomsk State University, 634050 Tomsk, Russia 
to the dilaton with an opposite profile. It is clear that the underlying action changes, and extra potential terms are generated depending on the dilaton field. These potential terms vanish only in the case of spin-1 modes. In Refs. 21] the sign of the dilaton was just changed without adding the corresponding potential terms, which is true only for total spin $J=1$ and not correct for higher spins. Here we agree with the criticism of Ref. [22].

In Refs. [6 9, 17, 19, 20] two equivalent versions of the soft-wall model for the study of the bound-state problem (hadronic mass spectrum) with a $\operatorname{positive} \exp (\varphi)[6,9,17]$ and a negative $\exp (-\varphi)$ [19, 20] dilaton exponential have been developed. These approaches are based on slightly different actions, which have the advantage of possible applications to mesons and baryons with adjustable quantum numbers of radial excitation, orbital and total angular momentum. In the study of the bound state problem both versions use effective actions quadratic in the bulk fields, which are equivalent due to a redefinition of the bulk field containing the dilaton. As a result both approaches arrive at the same equation of motion (EOM) for the mode dual to hadrons with specific quantum numbers. Therefore, when performing the matching of matrix elements in the soft-wall model and light-front QCD, a precise mapping of the bulk modes in the AdS fifth dimension to the hadron light-front wave functions can be performed (see details in Refs. [6 9, 24] ). The main objective of this paper is to demonstrate how to correctly derive the versions of the soft-wall model with different signs of the dilaton profile for the study of the hadronic mass spectrum. We also consider an extension of the soft-wall model to mesons and baryons with higher total angular momenta $J$.

When considering dynamical hadron quantities encoding a nontrivial momentum dependence (e.g. form factors, parton distributions) the sign of the dilaton profile becomes sufficient to guarantee fulfillment of the boundary conditions at $z \rightarrow \infty$. As it was shown in Ref. 22] the soft-wall model with a positive dilaton cannot be directly applied to the calculation of the bulk-to-boundary propagators of AdS fields by solving the corresponding differential equation in the $z$-dimension. The corresponding propagators $V\left(q^{2}, z\right)$ are dual to the external QCD currents and depend on the holographic coordinate $z$ and the transverse momentum squared $q^{2}$. In particular, the soft-wall model with a negative dilaton profile gives a bounded solution for the bulk-to-boundary propagator of the AdS vector field at $z \rightarrow \infty$. It also supplies the correct normalization $V(0, z)=1$ at $q^{2}=0$ in accordance with gauge invariance. The version with a positive profile, however, gives a divergent solution. This means that the positive version of the soft-wall model in its original form cannot be applied for the calculation of AdS bulk-to-boundary propagators and dynamical hadron properties. Therefore, the version with a negative dilaton can be applied without any additional modification (or restriction) to the calculation of both mass spectrum and dynamical properties of hadrons and we certainly prefer this realization of the soft-wall model.

\section{BOSONIC CASE}

\section{A. Scalar field}

First we demonstrate how to correctly derive the versions of soft-wall model with different signs of the dilaton profile for the study of hadronic mass spectrum. Afterwards we consider the fermionic field and the extension to higher values of the total angular momentum $J$. We consider the propagation of a scalar field $S(x, z)$ in $d+1$ dimensional AdS space. The AdS metric is specified by

$$
d s^{2}=g_{M N} d x^{M} d x^{N}=\eta_{a b} e^{2 A(z)} d x^{a} d x^{b}=e^{2 A(z)}\left(\eta_{\mu \nu} d x^{\mu} d x^{\nu}-d z^{2}\right), \quad \eta_{\mu \nu}=\operatorname{diag}(1,-1, \ldots,-1),
$$

where $M$ and $N=0,1, \cdots, d$ are the space-time (base manifold) indices, $a=(\mu, z)$ and $b=(\nu, z)$ are the local Lorentz (tangent) indices, $g_{M N}$ and $\eta_{a b}$ are curved and flat metric tensors, which are related by the vielbein $\epsilon_{M}^{a}(z)=e^{A(z)} \delta_{M}^{a}$ as $g_{M N}=\epsilon_{M}^{a} \epsilon_{N}^{b} \eta_{a b}$. Here $z$ is the holographic coordinate, $R$ is the AdS radius, and $g=\left|\operatorname{det} g_{M N}\right|=e^{2 A(z)(d+1)}$. In the following we restrict ourselves to a conformal-invariant metric with $A(z)=\log (R / z)$.

The actions for the scalar field $(J=0)$ with a positive or negative dilaton are [6, 9$]$

$$
S_{0}^{+}=\frac{1}{2} \int d^{d} x d z \sqrt{g} e^{\varphi(z)}\left[g^{M N} \partial_{M} S^{+}(x, z) \partial_{N} S^{+}(x, z)-\mu_{0}^{2} S^{+}(x, z) S^{+}(x, z)\right]
$$

and [19]

$$
S_{0}^{-}=\frac{1}{2} \int d^{d} x d z \sqrt{g} e^{-\varphi(z)}\left[g^{M N} \partial_{M} S^{-}(x, z) \partial_{N} S^{-}(x, z)-\left(\mu_{0}^{2}+\Delta V_{0}(z)\right) S^{-}(x, z) S^{-}(x, z)\right] .
$$

The superscripts + and - correspond to the cases of positive and negative dilaton, respectively, and $\varphi(z)=\kappa^{2} z^{2}$. The actions are equivalent to each other, which is obvious after performing the bulk field redefinition:

$$
S^{ \pm}(x, z)=e^{\mp \varphi(z)} S^{\mp}(x, z) .
$$


The difference between the two actions is absorbed in the effective potential $\Delta V_{0}(z)=e^{-2 A(z)} \Delta U_{0}(z)$, where

$$
\Delta U_{0}(z)=\varphi^{\prime \prime}(z)+(d-1) \varphi^{\prime}(z) A^{\prime}(z)
$$

with $F^{\prime}(z)=d F(z) / d z, F^{\prime \prime}(z)=d^{2} F(z) / d z^{2}$ and $F=\varphi, A$. The quantity $\mu_{0}^{2} R^{2}=\Delta(\Delta-d)$ is the bulk boson mass, where $\Delta$ is the dimension of the interpolating operator dual to the scalar bulk field. For the case of the bulk fields dual to the scalar mesons $\Delta=2+L$, where $L=\max \left|L_{z}\right|$ is the maximal value of the $z$-component of the quark orbital angular momentum in the LF wavefunction [6, 9]. In particular, we have the values $L=0$ for $J^{P}=0^{-}$states and $L=1$ for $J^{P}=0^{+}$states. Notice that $\Delta$ is identified with the twist $\tau$ of the two-parton states. Later we will show that $\tau$ for meson states is independent of the total angular momentum $J$, i.e. $\Delta_{J} \equiv \tau=2+L$. Notice that both actions have the correct conformal limit for $z \rightarrow 0$, where the dilaton field vanishes and conformal invariance is restored.

In a next step we modify the above forms of the action to obtain expressions which are more convenient in the applications. First, one can remove the dilaton field from the overall exponential by a specific redefinition of the bulk field $S^{ \pm}$with:

$$
S^{ \pm}(x, z)=e^{\mp \varphi(z) / 2} S(x, z)
$$

In terms of the field $S(x, z)$ the transformed action, which now is universal for both versions of the soft-wall model, reads 6 -9]:

$$
S_{0}=\frac{1}{2} \int d^{d} x d z \sqrt{g}\left[g^{M N} \partial_{M} S(x, z) \partial_{N} S(x, z)-\left(\mu_{0}^{2}+V_{0}(z)\right) S^{2}(x, z)\right]
$$

where $V_{0}(z)=e^{-2 A(z)} U_{0}(z)$ with the effective potential

$$
U_{0}(z)=\frac{1}{2} \varphi^{\prime \prime}(z)+\frac{1}{4}\left(\varphi^{\prime}(z)\right)^{2}+\frac{d-1}{2} \varphi^{\prime}(z) A^{\prime}(z) .
$$

The last expression is identical with the light-front effective potential found in Ref. [9] for $d=4, J=0$ [see Eq.(10)]:

$$
U_{0}(z)=\kappa^{4} z^{2}-2 \kappa^{2}
$$

With Lorentzian signature the action (17) is given by

$$
\begin{aligned}
S_{0} & =\frac{1}{2} \int d^{d} x d z e^{B_{0}(z)}\left[\partial_{\mu} S(x, z) \partial^{\mu} S(x, z)-\partial_{z} S(x, z) \partial_{z} S(x, z)-\left(e^{2 A(z)} \mu_{0}^{2}+U_{0}(z)\right) S^{2}(x, z)\right], \\
B_{0}(z) & =(d-1) A(z) .
\end{aligned}
$$

Now we use a Kaluza-Klein expansion

$$
S(x, z)=\sum_{n} S_{n}(x) \Phi_{n}(z)
$$

where $n$ is the radial quantum number, $S_{n}(x)$ is the tower of the Kaluza-Klein (KK) modes dual to scalar mesons and $\Phi_{n}$ are their extra-dimensional profiles (wave-functions). We assume a free propagation of the bulk field along the $d$ Poincaré coordinates with four-momentum $p$, and a constrained propagation along the $(d+1)$-th coordinate $z$ (due to confinement imposed by the dilaton field). On mass-shell $p^{2}=M_{n 0}^{2}$ the profiles $\Phi_{n}(z)$ obey the EOM

$$
\left[-\frac{d^{2}}{d z^{2}}-B_{0}^{\prime}(z) \frac{d}{d z}+e^{2 A(z)} \mu_{0}^{2}+U_{0}(z)\right] \Phi_{n}(z)=M_{n 0}^{2} \Phi_{n}(z) .
$$

Performing the substitution

$$
\Phi_{n}(z)=e^{-B_{0}(z) / 2} \phi_{n}(z)
$$

we derive the Schrödinger-type EOM for $\phi_{n}(z)$ :

$$
\left[-\frac{d^{2}}{d z^{2}}+\frac{4 L^{2}-1}{4 z^{2}}+U_{0}(z)\right] \phi_{n}(z)=M_{n 0}^{2} \phi_{n}(z)
$$


where

$$
\phi_{n}(z)=\sqrt{\frac{2 \Gamma(n+1)}{\Gamma(n+L+1)}} \kappa^{L+1} z^{L+1 / 2} e^{-\kappa^{2} z^{2} / 2} L_{n}^{L}\left(\kappa^{2} z^{2}\right)
$$

and

$$
M_{n 0}^{2}=4 \kappa^{2}\left(n+\frac{L}{2}\right)
$$

is the mass spectrum of the scalar field. Here we use the generalized Laguerre polynomials

$$
L_{n}^{m}(x)=\frac{x^{-m} e^{x}}{n !} \frac{d^{n}}{d x^{n}}\left(e^{-x} x^{m+n}\right) .
$$

Notice that the normalizable modes $\Phi_{n}(z)$ and $\phi_{n}(z)$ obey the following normalization conditions:

$$
\int_{0}^{\infty} d z e^{B_{0}(z)} \Phi_{m}(z) \Phi_{n}(z)=\int_{0}^{\infty} d z \phi_{m}(z) \phi_{n}(z)=\delta_{m n} .
$$

The mode $\Phi_{n}(z)$ has the correct behavior in both the ultraviolet (UV) and infrared (IR) limits:

$$
\Phi_{n}(z) \sim z^{2+L} \text { at small } z, \quad \Phi_{n}(z) \rightarrow 0 \text { at large } z .
$$

Using the KK expansion (11), the EOM (12) and the normalization condition (18) for the KK profiles $\Phi_{n}(z)$, the $d+1$-dimensional action for the bulk field reduces to a $d$-dimensional action for the scalar fields $S_{n}(x)$ dual to scalar mesons with masses $M_{n 0}$ :

$$
S_{0}^{(d)}=\frac{1}{2} \sum_{n} \int d^{d} x\left[\partial_{\mu} S_{n}(x) \partial^{\mu} S_{n}(x)-M_{n 0}^{2} S_{n}^{2}(x)\right] .
$$

This last equation is a manifestation of the gauge-gravity duality. In particular, it explicitly demonstrates that effective actions for conventional hadrons in $d$ dimensions can be generated from actions for bulk fields propagating in extra $d+1$ dimensions. The effect of the extra-dimension is encoded in the hadronic mass squared $M_{n}^{2}$, which is the solution of the Schrödinger equation (14) for the KK profile in extra dimension $\phi_{n}(z)$.

Another important conclusion is that we explicitly showed that correctly formulated soft-wall models with positive or negative dilaton profiles provide the same results, moving the dilaton field into the potential of the Schrödinger-type equation for the bound-state problem. One can also start with the action [see Eq. (77)] where the dilaton is hidden in the effective potential $V(z)$.

The next question concerns the choice of the $z$-dependence of the vielbein or the warping of the AdS metric, i.e. the choice of the function $A(z)$. As stressed before, the conformal-invariant limit is restricted to $A(z)=\log (R / z)$. What happens if the "conformal function" $A(z)$ is changed by an adjustable function - "warping function" $A_{W}(z)$, breaking the conformal invariance of the line element $d s^{2}$ ? It is easy to show that the Schrödinger-type EOM for $\phi_{n}(z)$ is modified by an extra potential term $W_{0}(z)$, which can be expressed in terms of $A_{W}(z)$ and $A(z)$ as

$$
W_{0}(z)=\mu^{2}\left[e^{2 A_{W}(z)}-e^{2 A(z)}\right]+\frac{(d-1)^{2}}{4}\left[A_{W}^{\prime}(z)-A^{\prime}(z)\right]^{2}+\frac{d-1}{2}\left[A_{W}^{\prime \prime}(z)-A^{\prime \prime}(z)\right] .
$$

It is clear that $W_{0}(z) \equiv 0$ for $A_{W}(z) \equiv A(z)$. Physical applications and consequences of the warping of the AdS metric have been studied in detail in Refs. [10 12. On the other hand, in order to guarantee that two types of soft-wall model ("dilaton" and "metric" ones) describe the same physics one should compensate such a correction by adding the corresponding potential in the actions: for the actions $S_{0}^{ \pm}$and for $S_{0}$ where the dilaton is hidden [see Eq. (10)] such correction is

$$
\Delta S_{0}^{W}=\frac{1}{2} \int d^{d} x d z \sqrt{g_{W}} e^{ \pm \varphi(z)} e^{-2 A_{W}(z)} W_{0}(z) S^{ \pm}(x, z) S^{ \pm}(x, z)=\frac{1}{2} \int d^{d} x d z e^{B_{0}(z)} W_{0}(z) S^{2}(x, z),
$$

where $g_{W}=e^{2 A_{W}(z)(d+1)}$ and $S^{ \pm}(x, z)=e^{\mp \varphi(z) / 2+\left(A(z)-A_{W}(z)\right)(d-1) / 2} S(x, z)$. 


\section{B. Vector field}

In the case of a vector bulk field $V_{M}(x, z)$ the actions with positive and negative dilaton are

$$
S_{1}^{+}=\frac{1}{2} \int d^{d} x d z \sqrt{g} e^{\varphi(z)}\left[-\frac{1}{2} g^{M_{1} M_{2}} g^{N_{1} N_{2}} V_{M_{1} N_{1}}^{+}(x, z) V_{M_{2} N_{2}}^{+}(x, z)+\left(\mu_{1}^{2}+\Delta V_{1}(z)\right) g^{M N} V_{M}^{+}(x, z) V_{N}^{+}(x, z)\right]
$$

and

$$
S_{1}^{-}=\frac{1}{2} \int d^{d} x d z \sqrt{g} e^{-\varphi(z)}\left[-\frac{1}{2} g^{M_{1} M_{2}} g^{N_{1} N_{2}} V_{M_{1} N_{1}}^{-}(x, z) V_{M_{2} N_{2}}^{-}(x, z)+\mu_{1}^{2} g^{M N} V_{M}^{-}(x, z) V_{N}^{-}(x, z)\right],
$$

where $V_{M N}=\partial_{M} V_{N}-\partial_{N} V_{M}$ is the stress tensor of the vector field. As in case of the scalar field, there exists the bulk field redefinition $V_{M}^{ \pm}=e^{\mp \varphi(z)} V_{M}^{\mp}$, making the two actions equivalent to each other. The mass of the vector bulk field is given by

$$
\mu_{1}^{2} R^{2}=\mu_{0}^{2} R^{2}+d-1=(\Delta-1)(\Delta+1-d)
$$

The difference between the two actions is absorbed in the effective potential $\Delta V_{1}(z)=e^{-2 A(z)} \Delta U_{1}(z)$, where

$$
\Delta U_{1}(z)=-\varphi^{\prime \prime}(z)+(3-d) \varphi^{\prime}(z) A^{\prime}(z) .
$$

Notice that $\Delta U_{1}(z) \equiv 0$ for $d=4, A(z)=\log (R / z)$ and $\varphi(z)=\kappa^{2} z^{2}$. Again, as in the scalar field case, one can remove the dilaton field from the overall exponential by a specific redefinition of the bulk field (6):

$$
S_{1}=\frac{1}{2} \int d^{d} x d z \sqrt{g}\left[-\frac{1}{2} g^{M_{1} M_{2}} g^{N_{1} N_{2}} V_{M_{1} N_{1}}(x, z) V_{M_{2} N_{2}}(x, z)+\left(\mu_{1}^{2}+V_{1}(z)\right) g^{M N} V_{M}(x, z) V_{N}(x, z)\right]
$$

where $V_{1}(z)=e^{-2 A(z)} U_{1}(z)$ and with the effective potential

$$
U_{1}(z)=-\frac{1}{2} \varphi^{\prime \prime}(z)+\frac{1}{4}\left(\varphi^{\prime}(z)\right)^{2}+\frac{3-d}{2} \varphi^{\prime}(z) A^{\prime}(z) .
$$

This last expression is identical with the light-front effective potential found in Ref. [9] for $d=4, J=1$ [see Eq.(10)]:

$$
U_{1}(z)=\kappa^{4} z^{2} .
$$

Using standard algebra and restricting ourselves to the axial gauge $V_{z}(x, z)=0$, we write down the action in terms of fields with Lorentz indices:

$$
\begin{aligned}
S_{1} & =\frac{1}{2} \int d^{d} x d z e^{B_{1}(z)}\left[-\frac{1}{2} V_{\mu \nu}(x, z) V^{\mu \nu}(x, z)+\partial_{z} V_{\mu}(x, z) \partial_{z} V^{\mu}(x, z)+\left(e^{2 A(z)} \mu_{1}^{2}+U_{1}(z)\right) V_{\mu}(x, z) V^{\mu}(x, z)\right], \\
B_{1}(z) & =B_{0}(z)-2 A(z)=(d-3) A(z) .
\end{aligned}
$$

It is convenient to rescale the vector fields by the boost (total angular momentum) factor $e^{A(z)}$ as $V_{\mu}(x, z) \rightarrow$ $e^{A(z)} V_{\mu}(x, z)$. For higher-spin states the boost factor is $e^{J A(z)}$ (see next subsection). Then the action takes the form

$$
S_{1}=\frac{1}{2} \int d^{d} x d z e^{B_{0}(z)}\left[-\frac{1}{2} V_{\mu \nu}(x, z) V^{\mu \nu}(x, z)+\partial_{z} V_{\mu}(x, z) \partial_{z} V^{\mu}(x, z)+\left(e^{2 A(z)} \mu_{0}^{2}+U_{1}(z)\right) V_{\mu}(x, z) V^{\mu}(x, z)\right] .
$$

We restrict to the transverse components $V^{\mu \perp}\left(\partial_{\mu} V^{\mu \perp}=0\right)$ and again use the KK expansion

$$
V^{\mu \perp}(x, z)=\sum_{n} V_{n}^{\mu}(x) \Phi_{n}(z)
$$

where $V_{n}^{\mu \perp}$ is the tower of the KK modes dual to vector mesons and $\Phi_{n}$ are their extra-dimensional profiles (wavefunctions). These coincide with the profiles of scalar mesons, i.e. are independent on total angular momentum $J$. In the case of vector mesons the EOM for the profile function $\Phi_{n}$ is given by

$$
\left[-\frac{d^{2}}{d z^{2}}-B_{0}^{\prime}(z) \frac{d}{d z}+e^{2 A(z)} \mu_{0}^{2}+U_{1}(z)\right] \Phi_{n}(z)=M_{n 1}^{2} \Phi_{n}(z) .
$$


Performing the substitution

$$
\Phi_{n}(z)=e^{-B_{0}(z) / 2} \phi_{n}(z)
$$

we derive the Schrödinger-type $\mathrm{EOM}$ for $\phi_{n}(z)$ :

$$
\left[-\frac{d^{2}}{d z^{2}}+\frac{4 L^{2}-1}{4 z^{2}}+U_{0}(z)\right] \phi_{n}(z)=M_{n 1}^{2} \phi_{n}(z)
$$

where

$$
M_{n 1}^{2}=4 \kappa^{2}\left(n+\frac{L}{2}+\frac{1}{2}\right) .
$$

One can see that the EOMs for $\Phi_{n}$ and $\phi_{n}$ in the case of vector mesons are different from the analogous EOMs for scalar mesons by the effective dilaton potential $U_{1}(z)=U_{0}(z)+2 \kappa^{2}$ for $\varphi(z)=\kappa^{2} z^{2}$ and $A(z)=\log (R / z)$, which generates the shift in the mass spectrum: $M_{n 1}^{2}=M_{n 0}^{2}+2 \kappa^{2}$.

As in the case of scalar mesons (using the KK expansion, EOM for the wave functions) we derive the d-dimensional action for the vector mesons with masses $M_{n 1}^{2}$ from the higher-dimensional $d+1$ action in terms of their holographic analogues:

$$
S_{1}^{(d)}=\sum_{n} \int d^{d} x\left[-\frac{1}{4} V_{\mu \nu, n}(x) V_{n}^{\mu \nu}(x)+\frac{M_{n 1}^{2}}{2} V_{\mu, n}(x) V_{n}^{\mu}(x)\right] .
$$

Notice that a possible warping of the metric gives the following correction to the effective potential

$$
W_{1}(z)=\mu^{2}\left[e^{2 A_{W}(z)}-e^{2 A(z)}\right]+\frac{(d-3)^{2}}{4}\left[A_{W}^{\prime}(z)-A^{\prime}(z)\right]^{2}+\frac{d-3}{2}\left[A_{W}^{\prime \prime}(z)-A^{\prime \prime}(z)\right] .
$$

Again in analogy with scalar mesons, such warping can be compensated by adding the corresponding potential in the action for the vector bulk field.

Finally, we would like to mention the results of Ref. [22], where the sign of the dilaton field was checked in order to fulfil different constraints going beyond the bound-state problem, e.g. absence of a massless state in the vector channel and a related problem $-q^{2}$ behavior of the vector-vector correlator function $\Pi_{V}\left(-q^{2}\right)$ consistent with QCD, correct normalization at $q^{2}=0$ of the vector bulk-to-boundary propagator corresponding to the electromagnetic current. The conclusion was that this requirement fix the sign of the dilaton profile: it should be negative (in our notations). It was shown there that the bulk-to-boundary propagator of the vector field $V(q, z)$ satisfying all criteria is given by [22]:

$$
V(q, z)=\Gamma\left(1-\frac{q^{2}}{4 \kappa^{2}}\right) U\left(-\frac{q^{2}}{4 \kappa^{2}}, 0, \kappa^{2} z^{2}\right) .
$$

This is related to the Fourier transform of the transverse massless $\left(\mu_{1}=0\right)$ vector bulk field

$$
V_{\mu \perp}^{-}(x, z)=\int \frac{d^{d} q}{(2 \pi)^{d}} e^{-i q x} V_{\mu}(q) V(q, z)
$$

entering the action with a negative dilaton profile:

$$
S_{1}^{-}=-\frac{1}{4} \int d^{d} x d z \sqrt{g} e^{-\varphi(z)} g^{M_{1} M_{2}} g^{N_{1} N_{2}} V_{M_{1} N_{1}}^{-}(x, z) V_{M_{2} N_{2}}^{-}(x, z)
$$

and obeying the following EOM:

$$
\partial_{z}\left(\frac{e^{-\varphi(z)}}{z} \partial_{z} V(q, z)\right)+q^{2} \frac{e^{-\varphi(z)}}{z} V(q, z)=0 .
$$

In other words, the correctly defined bulk-to-boundary propagator of the vector field $V(q, z)$ is identified with the one obtained in the version with the negative dilaton. Let us remind that this is different from the bound-state problem where the KK profile $\Phi_{n}(z)$ is independent on the dilaton sign. Therefore, the version with a negative dilaton can be applied without any restriction for both bound state and scattering problem and we certainly prefer this realization of 
soft-wall model. For illustration we remind the result for the pion electromagnetic form factor $F_{\pi}\left(Q^{2}\right)$ in the Euclidean region calculated with negative dilaton profile [7, 16]:

$$
F_{\pi}\left(Q^{2}\right)=\int_{0}^{\infty} \frac{d z}{z^{3}} e^{-\varphi(z)} V(Q, z) \Phi_{0}^{2}(z)=\frac{1}{1+\frac{Q^{2}}{4 \kappa^{2}}}
$$

where $\Phi_{0}(z)=\kappa z^{2} \sqrt{2}$ is the wave function for pion with $n=L=0$ and $V(Q, z)$ is the holographic analogue of electromagnetic field given by (39). It is clear that the change of the sign of the dilaton profile leads to divergence of the pion form factor. The similar situations is for the $\rho$-meson form factor [13] and for the nucleon form factors [18, 20].

\section{Higher $J$ boson fields}

In this section we consider bulk boson fields with higher values of $J \geq 2$. This problem, in the context of hard- and soft-wall models, has been considered before in Refs. [3, 5- 9, 11, 19]. In particular, it was shown that the soft-wall model reproduces the Regge-behavior of the mesonic mass spectrum $M_{n J}^{2} \sim n+J$. Here, extending our results for scalar and vector fields, we show that the bound-state problem is independent on the sign of the dilaton profile.

We describe a bosonic spin- $J$ field $\Phi_{M_{1} \cdots M_{J}}(x, z)$ by a symmetric, traceless tensor, satisfying the conditions

$$
\nabla^{M_{1}} \Phi_{M_{1} M_{2} \cdots M_{J}}=0, \quad g^{M_{1} M_{2}} \Phi_{M_{1} M_{2} \cdots M_{J}}=0 .
$$

The actions for the bulk field $\Phi_{J}$ with positive and negative dilatons are [6-9]

$$
\begin{aligned}
S_{J}^{+} & =\frac{(-)^{J}}{2} \int d^{d} x d z \sqrt{g} e^{\varphi(z)}\left[g^{M N} g^{M_{1} N_{1}} \cdots g^{M_{J} N_{J}} \nabla_{M} \Phi_{M_{1} \cdots M_{J}}^{+}(x, z) \nabla_{N^{N}} \Phi_{N_{1} \cdots N_{J}}^{+}(x, z)\right. \\
& \left.-\mu_{J}^{2} g^{M_{1} N_{1}} \cdots g^{M_{J} N_{J}} \Phi_{M_{1} \cdots M_{J}}^{+}(x, z) \Phi_{N_{1} \cdots N_{J}}^{+}(x, z)\right]
\end{aligned}
$$

and [19]

$$
\begin{aligned}
S_{J}^{-} & =\frac{(-)^{J}}{2} \int d^{d} x d z \sqrt{g} e^{-\varphi(z)}\left[g^{M N} g^{M_{1} N_{1}} \cdots g^{M_{J} N_{J}} \nabla_{M} \Phi_{M_{1} \cdots M_{J}}^{-}(x, z) \nabla_{N} \Phi_{N_{1} \cdots N_{J}}^{-}(x, z)\right. \\
& \left.-\left(\mu_{J}^{2}+\Delta V_{J}(z)\right) g^{M_{1} N_{1}} \cdots g^{M_{J} N_{J}} \Phi_{M_{1} \cdots M_{J}}^{-}(x, z) \Phi_{N_{1} \cdots N_{J}}^{-}(x, z)\right]
\end{aligned}
$$

Here $\nabla_{M}$ is the covariant derivative with respect to AdS coordinates, which is defined as

$$
\nabla_{M} \Phi_{M_{1} \cdots M_{J}}=\partial_{M} \Phi_{M_{1} \cdots M_{J}}-\Gamma_{M_{1} M}^{N} \Phi_{N M_{1} \cdots M_{J}}-\cdots-\Gamma_{M_{J} M}^{N} \Phi_{M_{1} \cdots M_{J-1} N}
$$

where

$$
\Gamma_{M N}^{K}=\frac{1}{2} g^{K L}\left(\frac{\partial g_{L M}}{\partial x^{N}}+\frac{\partial g_{L N}}{\partial x^{M}}-\frac{\partial g_{M N}}{\partial x^{L}}\right)
$$

is the affine connection.

In Refs. [6 9, 19] higher spin fields have been considered in a "weak gravity" approximation, restricting the analysis to flat metric and therefore identifying the covariant derivative with the normal derivative (i.e. neglecting the affine connection). First, we review these results and then consider the general case with covariant derivatives. In the following we call the scenario with normal derivatives scenario I and the scenario with covariant derivatives scenario II.

In scenario I the bulk mass is given by by $6-9,19]$

$$
\mu_{J}^{2} R^{2}=(\Delta-J)(\Delta+J-d)
$$

which is fixed by the behavior of bulk fields $\Phi_{J}$ near the ultraviolet boundary $z=0$. The potential $\Delta V_{J}(z)=$ $e^{-2 A(z)} \Delta U_{J}(z)$ is given by

$$
\Delta U_{J}(z)=\varphi^{\prime \prime}(z)+(d-1-2 J) \varphi^{\prime}(z) A^{\prime}(z) .
$$


Notice that both quantities $\mu_{J}^{2}$ and $\Delta U_{J}(z)$ are generalizations of the scalar $(J=0)$ and vector $(J=1)$ cases considered before. In particular, they are related to those for the scalar field as follows:

$$
\mu_{J}^{2} R^{2}=\mu_{0}^{2} R^{2}+J(d-J), \quad \Delta U_{J}(z)=\Delta U_{0}(z)-2 J \varphi^{\prime}(z) A^{\prime}(z)
$$

As before the two actions can be reduced to the action with a dilaton hidden in an additional potential term using the transformation

$$
\Phi_{M_{1} \cdots M_{J}}^{ \pm}(x, z)=e^{\mp \varphi(z) / 2} \Phi_{M_{1} \cdots M_{J}}(x, z) .
$$

Then the action takes the form

$$
\begin{aligned}
S_{J} & =\frac{(-)^{J}}{2} \int d^{d} x d z \sqrt{g}\left[g^{M N} g^{M_{1} N_{1}} \cdots g^{M_{J} N_{J}} \partial_{M} \Phi_{M_{1} \cdots M_{J}}(x, z) \partial_{N} \Phi_{N_{1} \cdots N_{J}}(x, z)\right. \\
& \left.-\left(\mu_{J}^{2}+V_{J}(z)\right) g^{M_{1} N_{1}} \cdots g^{M_{J} N_{J}} \Phi_{M_{1} \cdots M_{J}}(x, z) \Phi_{N_{1} \cdots N_{J}}(x, z)\right]
\end{aligned}
$$

where $V_{J}(z)=e^{-2 A(z)} U_{J}(z)$, and with the effective potential

$$
U_{J}(z)=\frac{1}{2} \varphi^{\prime \prime}(z)+\frac{1}{4}\left(\varphi^{\prime}(z)\right)^{2}+\frac{d-1-2 J}{2} \varphi^{\prime}(z) A^{\prime}(z) .
$$

This last expression is identical with the light-front effective potential found in Ref. [9] for $d=4$ and arbitrary $J$ [see Eq.(10)]:

$$
U_{J}(z)=\kappa^{4} z^{2}+2 \kappa^{2}(J-1)
$$

Using standard algebra and restricting to the axial gauge $\Phi_{\ldots z \ldots}(x, z)=0$, we write down the action in terms of fields with Lorentz indices:

$$
\begin{aligned}
S_{J} & =\frac{(-)^{J}}{2} \int d^{d} x d z e^{B_{J}(z)}\left[\partial_{\mu} \Phi_{\mu_{1} \cdots \mu_{J}}(x, z) \partial^{\mu} \Phi^{\mu_{1} \cdots \mu_{J}}(x, z)-\partial_{z} \Phi_{\mu_{1} \cdots \mu_{J}}(x, z) \partial_{z} \Phi^{\mu_{1} \cdots \mu_{J}}(x, z)\right. \\
& \left.-\left(e^{2 A(z)} \mu_{J}^{2}+U_{J}(z)\right) \Phi_{\mu_{1} \cdots \mu_{J}}(x, z) \Phi^{\mu_{1} \cdots \mu_{J}}(x, z)\right] \\
B_{J}(z) & =B_{0}(z)-2 J A(z)=(d-1-2 J) A(z) .
\end{aligned}
$$

It is convenient to rescale the fields by the boost (total angular momentum) factor $e^{J A(z)}$ as $\Phi_{\mu_{1} \cdots \mu_{J}}(x, z) \rightarrow$ $e^{J A(z)} \Phi_{\mu_{1} \cdots \mu_{J}}(x, z)$. Then the action takes the form

$$
\begin{aligned}
S_{J} & =\frac{(-)^{J}}{2} \int d^{d} x d z e^{B_{0}(z)}\left[\partial_{\mu} \Phi_{\mu_{1} \cdots \mu_{J}}(x, z) \partial^{\mu} \Phi^{\mu_{1} \cdots \mu_{J}}(x, z)-\partial_{z} \Phi_{\mu_{1} \cdots \mu_{J}}(x, z) \partial_{z} \Phi^{\mu_{1} \cdots \mu_{J}}(x, z)\right. \\
& \left.-\left(e^{2 A(z)} \mu_{0}^{2}+U_{J}(z)\right) \Phi_{\mu_{1} \cdots \mu_{J}}(x, z) \Phi^{\mu_{1} \cdots \mu_{J}}(x, z)\right]
\end{aligned}
$$

Doing the KK expansion

$$
\Phi^{\mu_{1} \cdots \mu_{J}}(x, z)=\sum_{n} \Phi_{n}^{\mu_{1} \cdots \mu_{J}}(x) \Phi_{n}(z)
$$

we derive an EOM for the profile function $\Phi_{n}$

$$
\left[-\frac{d^{2}}{d z^{2}}-B_{0}^{\prime}(z) \frac{d}{d z}+e^{2 A(z)} \mu_{0}^{2}+U_{J}(z)\right] \Phi_{n}(z)=M_{n J}^{2} \Phi_{n}(z)
$$

We stress again that the profile function $\Phi_{n}(z)$ is an universal function independent on $J$. All the $J$ dependence is hidden in the effective potential $U_{J}(z)$, which generates the corresponding $J$-dependence in the mass spectrum $M_{n J}^{2}$.

Performing the substitution

$$
\Phi_{n}(z)=e^{-B_{0}(z) / 2} \phi_{n}(z)
$$


we derive the Schrödinger-type EOM for $\phi_{n}(z)$ :

$$
\left[-\frac{d^{2}}{d z^{2}}+\frac{4 L^{2}-1}{4 z^{2}}+U_{J}(z)\right] \phi_{n}(z)=M_{n J}^{2} \phi_{n}(z)
$$

where

$$
M_{n J}^{2}=4 \kappa^{2}\left(n+\frac{L}{2}+\frac{J}{2}\right)
$$

is the mass spectrum of higher $J$ fields, which generalizes our results for scalar and vector fields, (16) and (36). At large values of $J$ or $L$ we reproduce the Regge behavior of the meson mass spectrum:

$$
M_{n J}^{2} \sim n+J .
$$

Finally, using the KK expansion and the EOMs for the wave functions, we derive the $d$-dimensional action for mesons with total angular momentum $J \geq 2$ and masses $M_{n J}^{2}$ :

$$
S_{J}^{(d)}=\frac{(-)^{J}}{2} \sum_{n} \int d^{d} x\left[\partial_{\mu} \Phi_{\mu_{1} \ldots \mu_{J}, n}(x) \partial^{\mu} \Phi_{n}^{\mu_{1} \ldots \mu_{J}}(x)-M_{n J}^{2} \Phi_{\mu_{1} \cdots \mu_{J}, n}(x) \Phi_{n}^{\mu_{1} \cdots \mu_{J}}(x)\right] .
$$

As in the cases of scalar and vector fields, the warping of the metric can give the following correction to the effective potential

$$
W_{J}(z)=\mu^{2}\left[e^{2 A_{W}(z)}-e^{2 A(z)}\right]+\frac{(d-1-2 J)^{2}}{4}\left[A_{W}^{\prime}(z)-A^{\prime}(z)\right]^{2}+\frac{d-1-2 J}{2}\left[A_{W}^{\prime \prime}(z)-A^{\prime \prime}(z)\right],
$$

which can be compensated by adding the following term in the effective actions $S_{J}^{ \pm}, S_{J}$ :

$$
\begin{aligned}
\Delta S_{J}^{W} & =\frac{(-)^{J}}{2} \int d^{d} x d z \sqrt{g_{W}} e^{ \pm \varphi(z)} e^{-2 A_{W}(z)} W_{J}(z) \Phi_{M_{1} \cdots M_{J}}^{ \pm}(x, z) \Phi^{M_{1} \cdots M_{J}, \pm}(x, z) \\
& =\frac{(-)^{J}}{2} \int d^{d} x d z e^{A_{W}(z)(d-1-2 J)} e^{ \pm \varphi(z)} W_{J}(z) \Phi_{\mu_{1} \cdots \mu_{J}}^{ \pm}(x, z) \Phi^{\mu_{1} \cdots \mu_{J}, \pm}(x, z) \\
& =\frac{(-)^{J}}{2} \int d^{d} x d z e^{B_{J}(z)} W_{J}(z) \Phi_{\mu_{1} \cdots \mu_{J}}(x, z) \Phi^{\mu_{1} \cdots \mu_{J}}(x, z)
\end{aligned}
$$

where $\Phi_{\mu_{1} \cdots \mu_{J}}^{ \pm}(x, z)=e^{\mp \varphi(z) / 2+\left(A(z)-A_{W}(z)\right)(d-1-2 J) / 2} \Phi_{\mu_{1} \cdots \mu_{J}}(x, z)$.

Now we consider scenario II, i.e. without truncation of covariant derivatives. The gauge-invariant actions for the totally symmetric higher spin boson fields have been considered e.g. in Refs. [27]. In this case the bulk mass is fixed by gauge invariance, and given by

$$
\mu_{J}^{2} R^{2}=J^{2}+J(d-5)+4-2 d .
$$

This mass leads to the following results for the scaling of the KK profiles: $\Phi_{n}(z) \sim z^{2+J}$ at $z \rightarrow 0$, and their masses are $M_{n J}^{2}=4 \kappa^{2}(n+J)$, which are acceptable only for the limiting cases $J=L$ and $J \rightarrow \infty$. Notice that the soft-wall actions (45) and (46) are obtained from gauge-invariant actions for totally symmetric higher spin boson fields [27] via the introduction of the dilaton field, which breaks conformal and gauge invariance. Therefore, it is not necessary to use the bulk mass given by Eq. (67). In particular, in order to get correct scaling for the KK profile $\Phi_{n}(z) \sim z^{2+L}$ and their masses given by Eq. (62), we should use

$$
\mu_{J}^{2} R^{2}=(\Delta-J)(\Delta+J-d)-J=\mu_{0}^{2} R^{2}+J(d-1-J) .
$$

In this case scenario II is fully equivalent to scenario I.

\section{FERMIONIC CASE}

\section{A. Spin $1 / 2$ fermions}

In the fermion case we first consider the low-lying $J=1 / 2$ modes $\Psi_{ \pm}(x, z)$ (here the index \pm corresponds again to scenarios with positive/negative dilaton profiles, respectively.) The actions with positive and negative dilaton read 
[17, 18, 20]

$$
\begin{aligned}
S_{1 / 2}^{ \pm} & =\int d^{d} x d z \sqrt{g} e^{ \pm \varphi(z)}\left[\frac{i}{2} \bar{\Psi}^{ \pm}(x, z) \epsilon_{a}^{M} \Gamma^{a} \mathcal{D}_{M} \Psi^{ \pm}(x, z)-\frac{i}{2}\left(\mathcal{D}_{M} \Psi^{ \pm}(x, z)\right)^{\dagger} \Gamma^{0} \epsilon_{a}^{M} \Gamma^{a} \Psi^{ \pm}(x, z)\right. \\
& \left.-\bar{\Psi}^{ \pm}(x, z)\left(\mu+V_{F}(z)\right) \Psi^{ \pm}(x, z)\right] .
\end{aligned}
$$

The quantity $\mu$ is the bulk fermion mass with $m=\mu R=\Delta-d / 2$, where $\Delta$ is the dimension of the baryon interpolating operator, which is related with the scaling dimension $\tau=3+L$ as $\Delta=\tau+1 / 2$. For $J=1 / 2$ we have two baryon multiplets $J^{P}=1 / 2^{+}$for $L=0$ and $J^{P}=1 / 2^{-}$for $L=1 . V_{F}(z)=\varphi(z) / R$ is the dilaton field dependent effective potential. Its presence is necessary due to the following reason. When the fermionic fields are rescaled

$$
\Psi^{ \pm}(x, z)=e^{\mp \varphi(z) / 2} \Psi(x, z),
$$

the dilaton field is removed from the overall exponential. In terms of the field $\Psi(x, z)$, the modified action, which is universal for both versions of the soft-wall model, reads as

$$
S_{1 / 2}=\int d^{d} x d z \sqrt{g}\left[\frac{i}{2} \bar{\Psi}(x, z) \epsilon_{a}^{M} \Gamma^{a} \mathcal{D}_{M} \Psi(x, z)-\frac{i}{2}\left(\mathcal{D}_{M} \Psi(x, z)\right)^{\dagger} \Gamma^{0} \epsilon_{a}^{M} \Gamma^{a} \Psi(x, z)-\bar{\Psi}(x, z)\left(\mu+V_{F}(z)\right) \Psi(x, z)\right] .
$$

The form of the potential $V_{F}(z)$ is constrained in order to get solutions of the EOMs for fermionic KK modes of left and right chirality, and the correct asymptotics of the nucleon electromagnetic form factors at large $Q^{2}$ [17, 18, 20]. Note that a possible warping of the AdS metric is irrelevant in the fermionic case, because it can be absorbed in the bulk fermion field upon their rescaling, just like we removed the exponential prefactor containing the dilaton field.

The covariant derivative for the spin $J=1 / 2$ field is obtained from the normal derivative by adding the spin connection term: $\omega_{M}^{a b}=A^{\prime}(z)\left(\delta_{z}^{a} \delta_{M}^{b}-\delta_{z}^{b} \delta_{M}^{a}\right)$ :

$$
\mathcal{D}_{M}=\partial_{M}-\frac{1}{8} \omega_{M}^{a b}\left[\Gamma_{a}, \Gamma_{b}\right]
$$

where $\Gamma^{a}=\left(\gamma^{\mu},-i \gamma^{5}\right)$ are the Dirac matrices.

The action in terms of the field with Lorentz indices is:

$$
S_{1 / 2}=\int d^{d} x d z e^{A(z) d} \bar{\Psi}(x, z)\left[i \not \partial+\gamma^{5} \partial_{z}+\frac{d}{2} A^{\prime}(z) \gamma^{5}-\frac{e^{A(z)}}{R}(m+\varphi(z))\right] \Psi(x, z),
$$

where the Dirac field satisfies the following EOM [17, 18, 20]:

$$
\left[i \not \partial+\gamma^{5} \partial_{z}+\frac{d}{2} A^{\prime}(z) \gamma^{5}-\frac{e^{A(z)}}{R}(m+\varphi(z))\right] \Psi(x, z)=0,
$$

with $\not \partial=\gamma^{\mu} \partial_{\mu}$. For the conformal-invariant metric with $A(z)=\log (R / z)$ we get

$$
\left[i z \not \partial+\gamma^{5} z \partial_{z}-\frac{d}{2} \gamma^{5}-m-\varphi(z)\right] \Psi(x, z)=0 .
$$

Based on these solutions the fermionic action should be extended by an extra term in the ultraviolet boundary (see details in Refs. [18, 26] ). Here we review the derivation of the EOMs for the KK modes dual to the left- and rightchirality spinors in the soft-wall model [17, 18, 20]. First we expand the fermion field in left- and right-chirality components:

$$
\Psi(x, z)=\Psi_{L}(x, z)+\Psi_{R}(x, z), \quad \Psi_{L / R}=\frac{1 \mp \gamma^{5}}{2} \Psi, \quad \gamma^{5} \Psi_{L / R}=\mp \Psi_{L / R} .
$$

Then we perform a KK expansion for the $\Psi_{L / R}(x, z)$ fields:

$$
\Psi_{L / R}(x, z)=\sum_{n} \Psi_{L / R}^{n}(x) F_{L / R}^{n}(z) .
$$

The KK modes $F_{L / R}^{n}(z)$ satisfy the two coupled one-dimensional EOMs [17, 18, 20]:

$$
\left[\partial_{z} \pm \frac{e^{A}}{R}(m+\varphi)+\frac{d}{2} A^{\prime}\right] F_{L / R}^{n}(z)= \pm M_{n} F_{R / L}^{n}(z),
$$


where $M_{n}$ is the mass of baryons with $J=1 / 2$. After straightforward algebra one can obtain decoupled EOMs:

$$
\left[-\partial_{z}^{2}-d A^{\prime} \partial_{z}+\frac{e^{2 A}}{R^{2}}(m+\varphi)^{2} \mp \frac{e^{A}}{R}\left(A^{\prime}(m+\varphi)+\varphi^{\prime}\right)-\frac{d^{2}}{4} A^{\prime 2}-\frac{d}{2} A^{\prime \prime}\right] F_{L / R}^{n}(z)=M_{n}^{2} F_{L / R}^{n}(z) .
$$

After the substitution

$$
F_{L / R}^{n}(z)=e^{-A(z) \cdot d / 2} f_{L / R}^{n}(z)
$$

we derive the Schrödinger-type EOM for $f_{L / R}^{n}(z)$

$$
\left[-\partial_{z}^{2}+\frac{e^{2 A}}{R^{2}}(m+\varphi)^{2} \mp \frac{e^{A}}{R}\left(A^{\prime}(m+\varphi)+\varphi^{\prime}\right)\right] f_{L / R}^{n}(z)=M_{n}^{2} f_{L / R}^{n}(z) .
$$

For $A(z)=\log (R / z)$ and $\varphi(z)=\kappa^{2} z^{2}$ we get

$$
\left[-\partial_{z}^{2}+\kappa^{4} z^{2}+2 \kappa^{2}\left(m \mp \frac{1}{2}\right)+\frac{m(m \pm 1)}{z^{2}}\right] f_{L / R}^{n}(z)=M_{n}^{2} f_{L / R}^{n}(z)
$$

where

$$
\begin{aligned}
& f_{L}^{n}(z)=\sqrt{\frac{2 \Gamma(n+1)}{\Gamma(n+m+3 / 2)}} \kappa^{m+3 / 2} z^{m+1} e^{-\kappa^{2} z^{2} / 2} L_{n}^{m+1 / 2}\left(\kappa^{2} z^{2}\right), \\
& f_{R}^{n}(z)=\sqrt{\frac{2 \Gamma(n+1)}{\Gamma(n+m+1 / 2)}} \kappa^{m+1 / 2} z^{m} e^{-\kappa^{2} z^{2} / 2} L_{n}^{m-1 / 2}\left(\kappa^{2} z^{2}\right)
\end{aligned}
$$

with $\int_{0}^{\infty} d z f_{L / R}^{m}(z) f_{L / R}^{n}(z)=\delta_{m n}$ and

$$
M_{n}^{2}=4 \kappa^{2}\left(n+m+\frac{1}{2}\right) .
$$

For $d=4$ we have $m=L+3 / 2$ and, therefore,

$$
\begin{aligned}
& f_{L}^{n}(z)=\sqrt{\frac{2 \Gamma(n+1)}{\Gamma(n+L+3)}} \kappa^{L+3} z^{L+5 / 2} e^{-\kappa^{2} z^{2} / 2} L_{n}^{L+2}\left(\kappa^{2} z^{2}\right) \\
& f_{R}^{n}(z)=\sqrt{\frac{2 \Gamma(n+1)}{\Gamma(n+L+2)}} \kappa^{L+2} z^{L+3 / 2} e^{-\kappa^{2} z^{2} / 2} L_{n}^{L+1}\left(\kappa^{2} z^{2}\right)
\end{aligned}
$$

and

$$
M_{n}^{2}=4 \kappa^{2}(n+L+2)
$$

where $L=0,1$ for $J=1 / 2$ fermions. One can see that the functions $F_{L / R}^{n}(z)$ have the correct scaling behavior for small $z$

$$
F_{L}^{n}(z) \sim z^{9 / 2+L}, \quad F_{R}^{n}(z) \sim z^{7 / 2+L}
$$

and vanish at large $z$ (confinement). As in the bosonic case, integration over the holographic coordinate $z$ gives a $d$-dimensional action for the fermion field $\Psi^{n}(x)=\Psi_{L}^{n}(x)+\Psi_{R}^{n}(x)$ :

$$
S_{1 / 2}^{(d)}=\sum_{n} \int d^{d} x \bar{\Psi}^{n}(x)\left[i \not \partial-M_{n}\right] \Psi^{n}(x) .
$$




\section{B. $J=3 / 2$ and higher $J \geq 5 / 2$ fermion fields}

Extension of our formalism to $J=3 / 2$ and higher spin states $J \geq 5 / 2$ is straightforward. In particular, for $J=3 / 2$ states we should construct the action in terms of spinor-vector fields $\Psi_{M}$, where $M$ is the AdS index:

$$
\begin{aligned}
S_{3 / 2}^{ \pm} & =\int d^{d} x d z \sqrt{g} e^{ \pm \varphi(z)} g^{K N}\left[\frac{i}{2} \bar{\Psi}_{K}^{ \pm}(x, z) \epsilon_{a}^{M} \Gamma^{a} \mathcal{D}_{M} \Psi_{N}^{ \pm}(x, z)-\frac{i}{2}\left(\mathcal{D}_{M} \Psi_{K}^{ \pm}(x, z)\right)^{\dagger} \Gamma^{0} \epsilon_{a}^{M} \Gamma^{a} \Psi_{N}^{ \pm}(x, z)\right. \\
& \left.-\bar{\Psi}_{K}^{ \pm}(x, z)\left(\mu+V_{F}(z)\right) \Psi_{N}^{ \pm}(x, z)\right]
\end{aligned}
$$

and $\mathcal{D}_{M}$ is the covariant derivative acting on the spinor-vector field $\Psi_{N}$ as

$$
\mathcal{D}_{M} \Psi_{N}=\partial_{M} \Psi_{N}-\Gamma_{M N}^{K} \Psi_{K}-\frac{1}{8} \omega_{M}^{a b}\left[\Gamma_{a}, \Gamma_{b}\right] \Psi_{N}
$$

Notice that the spin $\omega_{M}^{a b}$ and affine $\Gamma_{M N}^{K}$ connections are related as

$$
\omega_{M}^{a b}=\epsilon_{K}^{a}\left(\partial_{M} \epsilon^{K b}+\epsilon^{N b} \Gamma_{M N}^{K}\right)
$$

After removing the dilaton field from the exponential prefactor, doing the boost of the spin-vector field $\Psi_{M}(x, z) \rightarrow$ $e^{A(z)} \Psi_{M}(x, z)$, and restricting to the axial gauge $\Psi_{z}(x, z)=0$, we derive the action in terms of fields with Lorentz indices:

$$
S_{3 / 2}=\int d^{d} x d z e^{A(z) d} \bar{\Psi}^{\mu}(x, z)\left[i \not \partial+\gamma^{5} \partial_{z}+\frac{d}{2} A^{\prime}(z) \gamma^{5}-\frac{e^{A(z)}}{R}(m+\varphi(z))\right] \Psi_{\mu}(x, z) .
$$

Then we proceed in analogy with the $J=1 / 2$ case, and derive the same $L$ dependent and $J$ independent EOM, which is consistent with the results of Ref. [11].

Finally, the actions for higher spin $J \geq 5 / 2$ fermions with positive and negative dilaton are written as

$$
\begin{aligned}
S_{J}^{ \pm} & =\int d^{d} x d z \sqrt{g} e^{ \pm \varphi(z)} g^{K_{1} N_{1}} \cdots g^{K_{J-1 / 2} N_{J-1 / 2}}\left[\frac{i}{2} \bar{\Psi}_{K_{1} \cdots K_{J-1 / 2}}^{ \pm}(x, z) \epsilon_{a}^{M} \Gamma^{a} \mathcal{D}_{M} \Psi_{N_{1} \cdots N_{J-1 / 2}}^{ \pm}(x, z)\right. \\
& \left.-\frac{i}{2}\left(\mathcal{D}_{M} \Psi_{K_{1} \cdots K_{J-1 / 2}}^{ \pm}(x, z)\right)^{\dagger} \Gamma^{0} \epsilon_{a}^{M} \Gamma^{a} \Psi_{N_{1} \cdots N_{J-1 / 2}}^{ \pm}(x, z)-\bar{\Psi}_{K_{1} \cdots K_{J-1 / 2}}^{ \pm}(x, z)\left(\mu+V_{F}(z)\right) \Psi_{N_{1} \cdots N_{J-1 / 2}}^{ \pm}(x, z)\right]
\end{aligned}
$$

where the covariant derivative $\mathcal{D}_{M}$ acting on spin-tensor field $\Psi_{N_{1} \cdots N_{J-1 / 2}}^{ \pm}$is

$$
\begin{aligned}
\mathcal{D}_{M} \Psi_{N_{1} \cdots N_{J-1 / 2}} & =\partial_{M} \Psi_{N_{1} \cdots N_{J-1 / 2}}-\Gamma_{M N_{1}}^{K} \Psi_{K N_{2} \cdots N_{J-1 / 2}}-\cdots- \\
& -\Gamma_{M N_{J-1 / 2}}^{K} \Psi_{N_{1} \cdots N_{J-3 / 2} K}-\frac{1}{8} \omega_{M}^{a b}\left[\Gamma_{a}, \Gamma_{b}\right] \Psi_{N_{1} \cdots N_{J-1 / 2}}
\end{aligned}
$$

As before, for the $J=1 / 2,3 / 2$ cases we remove the dilaton field from the exponential prefactor, perform the boost of the spin-tensor field and restrict ourselves to the axial gauge. Then we derive the action in terms of fields with Lorentz indices:

$$
S_{J}=\int d^{d} x d z e^{A(z) d} \bar{\Psi}^{\mu_{1} \cdots \mu_{J-1 / 2}}(x, z)\left[i \not \partial+\gamma^{5} \partial_{z}+\frac{d}{2} A^{\prime}(z) \gamma^{5}-\frac{e^{A(z)}}{R}(m+\varphi(z))\right] \Psi_{\mu_{1} \cdots \mu_{J-1 / 2}}(x, z) .
$$

Next, after a straightforward algebra (including the KK expansion), we derive the same equation of motion for the KK profile and mass formula as for fermions with lower spins. The action for physical baryons with higher spins (the result for $J=3 / 2$ is straightforward) is written as

$$
S_{J}^{(d)}=\sum_{n} \int d^{d} x \bar{\Psi}^{\mu_{1} \cdots \mu_{J-1 / 2}, n}(x)\left[i \not \partial-M_{n}\right] \Psi_{\mu_{1} \cdots \mu_{J-1 / 2}}^{n}(x) .
$$

Therefore, the main difference between the bosonic and fermionic actions is that in the case of bosons the mass formula depends on the combination $(J+L) / 2$, while in the baryon case it depends only on $L$. Also, in the fermion case the dilaton prefactor and possible warping of conformal-invariant AdS metric can be easily absorbed in the field, without the generation of extra potential terms. 


\section{APPLICATIONS}

\section{A. Basic properties of pion and nucleon}

We consider several applications of our soft-wall model with negative dilaton profile. First, we display the predictions for basic properties of pions and nucleons with the same value of dilaton parameter $\kappa=350 \mathrm{MeV}$.

The masses of pion and nucleon are:

$$
M_{\pi}=0 \quad M_{N}=2 \sqrt{2} \kappa=990 \mathrm{MeV} .
$$

The pion decay constant is given by [9]:

$$
F_{\pi}=\frac{\sqrt{3}}{8} \kappa=76 \mathrm{MeV} .
$$

The electromagnetic radii of pion and nucleon are given by the expression (see details in Refs. 7, 16, 18, 20]):

$$
\begin{aligned}
\left\langle r^{2}\right\rangle^{\pi} & =\frac{12}{M_{N}^{2}}, \quad\left\langle r_{E}^{2}\right\rangle^{p}=\frac{147}{8 M_{N}^{2}}\left(1+\frac{13}{147} \mu_{p}\right), \quad\left\langle r_{E}^{2}\right\rangle^{n}=\frac{13}{8 M_{N}^{2}} \mu_{n}, \\
\left\langle r_{M}^{2}\right\rangle^{p} & =\frac{177}{8 M_{N}^{2}}\left(1-\frac{17}{177 \mu_{p}}\right), \quad\left\langle r_{M}^{2}\right\rangle^{n}=\frac{177}{8 M_{N}^{2}},
\end{aligned}
$$

where $\mu_{p}$ and $\mu_{n}$ are the magnetic moments of nucleons. Here, for convenience, we expressed the dilaton parameter through the nucleon mass. Using data for $\mu_{p}=2.793$ and $\mu_{n}=-1.913$, the results for the slopes compared rather well with data:

$$
\begin{aligned}
& \left\langle r^{2}\right\rangle^{\pi}=0.476 \mathrm{fm}^{2} \text { (our), } 0.452 \mathrm{fm}^{2} \text { (data), } \\
& \left\langle r_{E}^{2}\right\rangle^{p}=0.910 \mathrm{fm}^{2} \text { (our), } 0.766 \mathrm{fm}^{2} \text { (data), } \\
& \left\langle r_{E}^{2}\right\rangle^{n}=-0.123 \mathrm{fm}^{2} \text { (our), } \quad-0.116 \mathrm{fm}^{2} \text { (data), } \\
& \left\langle r_{M}^{2}\right\rangle^{p}=0.849 \mathrm{fm}^{2} \text { (our), } 0.731 \mathrm{fm}^{2} \text { (data), } \\
& \left\langle r_{M}^{2}\right\rangle^{n}=0.879 \mathrm{fm}^{2} \text { (our), } 0.762 \mathrm{fm}^{2} \text { (data) }
\end{aligned}
$$

Other important applications of our approach can be found in [19, 20]. In particular, in [19] we presented a detailed analysis of meson mass spectrum and decay constants. Moreover, in Ref. [20] we did the first calculation of nucleon generalized parton distributions in AdS/QCD.

\section{B. Baryon mass spectrum}

Here we present the application of our approach to the baryon spectrum. We remind that the baryon mass spectrum calculated in our formalism is specified by the radial quantum number $n$ and orbital angular momentum $L$.

$$
M_{n L}^{2}=4 \kappa^{2}(n+L+2)
$$

It means that the states with different spin $S=1 / 2$ and $S=3 / 2$ and fixed $L$ are degenerate. As is well-known, this degeneracy is removed by taking into account a hyperfine (HF) spin-spin interaction between quarks in the baryon, due to one-gluon exchange [28,31]:

$$
H_{\mathrm{hyp}}=\frac{1}{2} \sum_{i<j} \mathcal{H}_{i j} \vec{s}_{i} \cdot \vec{s}_{j} \delta^{3}\left(\vec{r}_{i}-\vec{r}_{j}\right)
$$

where $\vec{s}_{i}$ is the spin operator acting on the $i$-th quark. Here, $\mathcal{H}_{i j}$ is the two-body quark coupling, which includes a common color factor $-2 / 3$ and explicitly depends on the flavor of the constituent quarks through their masses $m_{i}$ and $m_{j}$ :

$$
\mathcal{H}_{i j} \sim \frac{2}{3} \frac{1}{m_{i} m_{j}}
$$


The use of $S U(6)$ spin-flavor wave functions for the ground-state baryons $B$ leads to simple relations between the matrix elements $\left\langle B\left|H_{\text {hyp }}\right| B\right\rangle$, which are the perturbative mass shifts due to the HF interaction.

Denoting the contribution from a non-strange quark pair as $\mathcal{H}_{q q}$ (and similarly for strange quarks with $q$ replaced by $s$ ), in the isospin limit the masses of the ground-state baryons are composed as [29]

$$
\begin{array}{ll}
M_{N}=3 E_{0}-\frac{3}{8} \mathcal{H}_{q q}, & M_{\Delta}=3 E_{0}+\frac{3}{8} \mathcal{H}_{q q} \\
M_{\Lambda}=2 E_{0}+E_{0}^{s}-\frac{3}{8} \mathcal{H}_{q q}, & M_{\Sigma^{*}}=2 E_{0}+E_{0}^{s}+\frac{1}{8} \mathcal{H}_{q q}+\frac{1}{4} \mathcal{H}_{q s} \\
M_{\Sigma}=2 E_{0}+E_{0}^{s}+\frac{1}{8} \mathcal{H}_{q q}-\frac{1}{2} \mathcal{H}_{q s}, & M_{\Xi^{*}}=E_{0}+2 E_{0}^{s}+\frac{1}{4} \mathcal{H}_{q s}+\frac{1}{8} \mathcal{H}_{s s} \\
M_{\Xi}=E_{0}+2 E_{0}^{s}-\frac{1}{2} \mathcal{H}_{q s}+\frac{1}{8} \mathcal{H}_{s s}, & M_{\Omega}=3 E_{0}^{s}+\frac{3}{8} \mathcal{H}_{s s}
\end{array}
$$

Here $E_{0}$ and $E_{0}^{s}$ are the single particle ground-state energies of the non-strange and strange quark, respectively. These mass formulas satisfy the Gell-Mann-Okubo mass relations

$$
M_{\Sigma}-M_{N}=\frac{1}{2}\left(M_{\Xi}-m_{N}\right)+\frac{3}{4}\left(M_{\Sigma}-M_{\Lambda}\right), \quad M_{\Sigma^{*}}-M_{\Delta}=M_{\Xi^{*}}-M_{\Sigma^{*}}=M_{\Omega}-M_{\Xi^{*}}
$$

providing a condition on the matrix elements of the residual interaction with $\mathcal{H}_{q q}-\mathcal{H}_{q s} \simeq \mathcal{H}_{q s}-\mathcal{H}_{s s}$. With the choice $E_{0}^{s}-E_{0} \simeq 180 \mathrm{MeV}, \mathcal{H}_{q q} \simeq 400 \mathrm{MeV}$ and $\mathcal{H}_{q q}-\mathcal{H}_{q s} \simeq \mathcal{H}_{q s}-\mathcal{H}_{s s} \simeq 150 \mathrm{MeV}$, all the observed mass differences can be approximately reproduced. Another important piece which contributes to the baryon masses is the meson cloud (MC) induced mass shift, due to the interaction between quarks and pseudoscalar $(\pi, K, \eta)$ meson fields. Such contribution was evaluated using different chiral quark models (see e.g. detailed discussion in Ref. [30]). It was proved that $\mathrm{MC}$ contribution is negative and is similar on magnitude for the octet and decuplet states.

Here we suggest a phenomenological formula for the square of the baryon mass, treating the HF splitting and MC corrections perturbatively (it means that we restrict to the first-order in such effects). In particular, we assume the following conjecture for the light baryon masses, including $\mathrm{HF}\left(\delta M_{S}^{2}\right)$ and $\mathrm{MC}\left(\delta M_{C}^{2}\right)$ corrections:

$$
M_{B}^{2}=M_{n L}^{2}+\delta M_{S}^{2}+\delta M_{C}^{2}=4 \kappa^{2}\left(n+L+\alpha_{B} S+2-\alpha_{B}-\beta_{B}\right) .
$$

where $M_{n L}^{2}$ is the leading term (103) predicted by the soft-wall model, while the terms $\delta M_{\mathrm{S}}^{2}$ and $\delta M^{2}$ are:

$$
\delta M_{S}^{2}=4 \alpha_{B} \kappa^{2}(S-1), \quad \delta M_{C}^{2}=-4 \beta_{B} \kappa^{2} .
$$

Here $S=1 / 2$ or $3 / 2$ is the internal spin, $\alpha_{B}$ and $\beta_{B}$ are free parameters. Note that at $\alpha_{B}=1 / 2$ and $\beta_{B}=3 / 4$ our empirical formula coincides with the one previously derived by Brodsky and de Téramond [8], where they subtracted the constant term from the light-front Hamiltonian matched to AdS/QCD Hamiltonian:

$$
M_{B}^{2}=4 \kappa^{2}(n+L+S / 2+3 / 4) .
$$

Formula (110) was derived in limit of SU(3) flavor invariance. Lets go beyond this and include $\mathrm{SU}(3)$ breaking effects caused by the strange-nonstrange quark mass difference $\delta_{s}=m_{s}-m_{q}$. Taking into account the SU(6) algebra for HF couplings [see Eq. (106)] and SU(3) shifts $\delta_{B}$ of the single particle ground-state energies in the baryon [see Eq. (106)] we extend the master formula as

$$
M_{B}=2 \kappa \sqrt{n+L+2+\alpha_{B}(S-1)-\beta_{B}}+\delta_{B}
$$

where

$$
\begin{aligned}
& \alpha_{N}=\alpha_{\Lambda}=\alpha_{D}=\alpha, \quad \alpha_{\Sigma}=\frac{4 r_{s}-1}{3} \alpha, \quad \alpha_{\Xi}=\frac{\left(4-r_{s}\right) r_{s}}{3} \alpha \\
& \alpha_{\Sigma^{*}}=\frac{1+2 r_{s}}{3} \alpha, \quad \alpha_{\Xi^{*}}=\frac{\left(2+r_{s}\right) r_{s}}{3} \alpha, \quad \alpha_{\Omega}=r_{s}^{2} \alpha
\end{aligned}
$$

and

$$
\delta_{N}=\delta_{\Delta}=0, \quad \delta_{\Lambda}=\delta_{\Sigma}=\delta_{\Sigma^{*}}=\delta_{s}, \quad \delta_{\Xi}=\delta_{\Xi^{*}}=2 \delta_{s}, \quad \delta_{\Omega}=3 \delta_{s} .
$$

Here $r_{s}=m_{q} / m_{s}$. The $\mathrm{SU}(3)$ limit corresponds to the conditions $r_{s}=1, \delta_{s}=0$. For $\mathrm{MC}$ correction we use the universal parameter $\beta_{B}=\beta$.

In our calculation of baryon masses we use 7 free parameters: the dilaton profile parameter $\kappa$, the couplings $\alpha$ and $\beta$, the set of constituent quark masses $m_{q}=m_{u}=m_{d}, m_{s}, m_{c}$ and $m_{b}$. The parameter $\kappa$ fixes the slopes of the 
baryon mass trajectories. Using data for excited light baryons we fix the value to $\kappa=500 \mathrm{MeV}$, which is also in accordance with the analysis of Ref. [8]. Notice that this value is also close to the value of $\kappa=550 \mathrm{MeV}$ found in the study of meson properties in [19]. The free parameters $\alpha$ and $\beta$ are fixed by the nucleon and $\Delta(1232)$ isobar masses, using two constraints from the baryon mass formula (111):

$$
M_{N}^{2}=4 \kappa^{2}(2-\alpha / 2-\beta), \quad M_{\Delta}^{2}=4 \kappa^{2}(2+\alpha / 2-\beta),
$$

which lead to

$$
\alpha=\frac{M_{\Delta}^{2}-M_{N}^{2}}{4 \kappa^{2}}, \quad \beta=2-\frac{M_{\Delta}^{2}+M_{N}^{2}}{8 \kappa^{2}} .
$$

For $\kappa=500 \mathrm{MeV}$ we get $\alpha=0.636$ and $\beta=0.800$. The light quark masses $m_{q}=400 \mathrm{MeV}$ and $m_{s}=575 \mathrm{MeV}$ are fixed from data for ground-state masses of light hyperons. Finally, the masses of the charm and bottom quarks $m_{c}=1.747 \mathrm{GeV}$ and $m_{b}=5.081 \mathrm{GeV}$ are fixed from data on the $\Lambda_{c}(2286)$ and $\Lambda_{b}(5620)$ masses. Finally we consider the set of parameters

$$
\begin{aligned}
& \kappa=500 \mathrm{MeV}, \quad \alpha=0.636, \quad \beta=0.800, \\
& m_{q}=400 \mathrm{MeV}, \quad m_{s}=575 \mathrm{MeV}, \quad m_{s}=1.747 \mathrm{GeV}, \quad m_{s}=5.081 \mathrm{GeV} .
\end{aligned}
$$

as the best fit. We want to stress that in our approach we consider constituent quarks. In particular, in the light sector the masses of the $u, d, s$ quarks encode spontaneous breaking of chiral symmetry and do not vanish in the chiral limit (when the corresponding current quark masses vanish). Moreover, hyperfine splitting effects must be described in terms of constituent quark masses (the use of current quark masses leads to a divergence of the hyperfine splitting in the chiral limit). On the other hand, in the context of chiral quark models (see e.g. discussion in Ref. [32]) it is possible to establish a relation between the constituent and the current quark masses in the form of a chiral expansion, which is consistent with low-energy theorems of QCD. It was shown in 32] that realistic values for the current masses of $u, d$ and $s$ quarks (found in lattice QCD and fixed in chiral perturbation theory) correspond to the following values of the constituent quark masses $m_{q}=420 \mathrm{MeV}$ (for $u, d$-quarks) and $m_{s}=590 \mathrm{MeV}$ (for the $s$-quark). These values compare well with the parameters used in our soft-wall model $\left(m_{q}=400 \mathrm{MeV}\right.$ and $\left.m_{s}=575 \mathrm{MeV}\right)$. In case of heavy quarks we use constituent quark masses which are a bit larger than the values quoted by the Particle Data Group [33]. The present set of parameters $\left(\kappa, m_{q}, m_{s}, m_{c}, m_{b}\right)$ is close to those used in the analysis of meson physics [19]:

$$
\kappa=550 \mathrm{MeV}, \quad m_{q}=420 \mathrm{MeV}, \quad m_{s}=570 \mathrm{MeV}, \quad m_{c}=1.6 \mathrm{GeV}, \quad m_{b}=4.8 \mathrm{GeV} .
$$

Also the set of constituent quark masses is very close to the one used in the Lorentz covariant three-quark model in a detailed description of exclusive strong, electromagnetic and weak decays of light and heavy baryons [37, 38]:

$$
m_{q}=420 \mathrm{MeV}, \quad m_{s}=570 \mathrm{MeV}, \quad m_{c}=1.7 \mathrm{GeV}, \quad m_{b}=5.2 \mathrm{GeV} .
$$

For completeness we indicate the relative error in the calculation of light and single-heavy baryon masses defined as $\delta_{\text {err }}=\left|\left(M_{B}^{\exp }-M_{B}^{\text {th }}\right) / M_{B}^{\exp }\right| \cdot 100 \%$, where $M_{B}^{\exp }$ and $M_{B}^{\text {th }}$ are the central values of the respective experimental and theoretical baryon masses. For the ground states (24 states) we get $\delta_{\text {err }} \leq 1 \%$, while for the excited ones (60 states) we have $\delta_{\text {err }} \leq 5 \%$.

With the set of parameters given in Eq. (116) we indicate our results for the light and heavy baryon masses in the following Tables I-VII. Table I contains the results for the ground states of light baryons. In Tables II and III we display our results for the excited states of the $N, \Delta, \Lambda, \Sigma$ and $\Sigma^{*}$ families, with different values of $n$ and $L$, and compare them with available data. In Tables IV and V we present a detailed classification of single, double and triple heavy baryons and results for their mass spectrum. Also we specify the HF couplings $\alpha_{B}$ (i.e. the ratio $\alpha_{B} / \alpha$ ). We introduced the notations $r_{c}=m_{q} / m_{c}$ and $r_{b}=m_{q} / m_{b}$. Note, we consider the mass spectrum of heavy baryons containing a single, two and three heavy $b$ or $c$ quarks using the master formula (111) with the same parameters of $\alpha$, $\beta, m_{q}, m_{s}$ and $\kappa$. We compare our results with available data 33] or with prediction of QCD motivated relativistic quark models $34-36]$. Single- and double-heavy baryons are classified by the set of quantum numbers $\left(J^{P}, S_{d}\right)$, where $J^{P}$ is the spin-parity of the baryon state and $S_{d}$ is the spin of the light or heavy diquark, respectively (see details in Ref. [37]). There are two types of light and heavy diquarks - those with $S_{d}=0$ (antisymmetric spin configuration $\left[q_{1} q_{2}\right]$ ) and those with $S_{d}=1$ (symmetric spin configuration $\left\{q_{1} q_{2}\right\}$ ). Accordingly there are two $J^{P}=1 / 2^{+}$singleand double-heavy baryon states. We follow the standard convention and attach a prime to the $S_{d}=1$ states whereas the $S_{d}=0$ states are unprimed in the case of single-heavy baryons, and vice versa in the case of double-heavy baryons — we attach a prime to the $S_{d}=0$ states whereas the $S_{d}=1$ states are unprimed. Finally, in Tables VI and VII we present our results for the mass spectrum of excited states of single-heavy baryons $\Lambda_{Q}, \Sigma_{Q}^{(*)}, \Xi_{Q}^{(*)}$ and $\Omega_{Q}^{(*)}$ and compare it with the recent prediction of relativistic quark-diquark model [35]. 


\section{CONCLUSIONS}

We performed a systematic analysis of extra-dimensional actions for bosons and fermions, which give rise to actions for observable hadrons. Masses of these hadrons are calculated analytically from Schrödinger type equations of motion with a potential which provides confinement of the Kaluza-Klein $($ KK) modes in extra $(d+1)$ dimension. The tower of KK modes with radial quantum number $n$ and total angular momentum $J$ has direct correspondence to realistic mesons and baryons living in $d$ dimensions. For such correspondence the sign of the dilaton profile is irrelevant, because the exponential prefactor containing the dilaton is finally absorbed in the bulk fields. On the other hand, the sign of the dilaton profile becomes important for the definition/calculation of the bulk-to-boundary propagator - i.e. the Green function describing the evolution of the bulk field from inside of AdS space to its ultraviolet boundary. The corresponding sign should be negative in order to fulfill certain constraints. It was discussed recently in Refs. 22. As application of our approach we presented detailed analysis of mass spectrum of light and heavy baryons.

\section{Acknowledgments}

The authors thank Stan Brodsky and Guy de Téramond for useful discussions and remarks. This work was supported by Federal Targeted Program "Scientific and scientific-pedagogical personnel of innovative Russia" Contract No. 02.740.11.0238, by FONDECYT (Chile) under Grant No. 1100287. V. E. L. would like to thank Departamento de Física y Centro Científico Tecnológico de Valparaíso (CCTVal), Universidad Técnica Federico Santa María, Valparaíso, Chile for warm hospitality. A. V. acknowledges the financial support from FONDECYT (Chile) Grant No. 3100028.

[1] J. M. Maldacena, Adv. Theor. Math. Phys. 2, 231 (1998) [Int. J. Theor. Phys. 38, 1113 (1999)] arXiv:hep-th/9711200]; S. S. Gubser, I. R. Klebanov and A. M. Polyakov, Phys. Lett. B 428, 105 (1998) arXiv:hep-th/9802109; E. Witten, Adv. Theor. Math. Phys. 2, 253 (1998) arXiv:hep-th/9802150.

[2] J. Polchinski and M. J. Strassler, Phys. Rev. Lett. 88, 031601 (2002) arXiv:hep-th/0109174]; H. Boschi-Filho and N. R. F. Braga, JHEP 0305, 009 (2003) arXiv:hep-th/0212207; G. F. de Teramond and S. J. Brodsky, Phys. Rev. Lett. 94, 201601 (2005) arXiv:hep-th/0501022 ; Phys. Rev. Lett. 102, 081601 (2009) arXiv:0809.4899 [hep-ph]]; J. Erlich, E. Katz, D. T. Son and M. A. Stephanov, Phys. Rev. Lett. 95, 261602 (2005) arXiv:hep-ph/0501128; L. Da Rold and A. Pomarol, Nucl. Phys. B 721, 79 (2005) arXiv:hep-ph/0501218; K. Ghoroku, N. Maru, M. Tachibana and M. Yahiro, Phys. Lett. B 633, 602 (2006) arXiv:hep-ph/0510334.

[3] E. Katz, A. Lewandowski and M. D. Schwartz, Phys. Rev. D 74, 086004 (2006) arXiv:hep-ph/0510388].

[4] H. R. Grigoryan and A. V. Radyushkin, Phys. Lett. B 650, 421 (2007) arXiv:hep-ph/0703069; Phys. Rev. D 76, 115007 (2007) arXiv:0709.0500 [hep-ph]].

[5] A. Karch, E. Katz, D. T. Son and M. A. Stephanov, Phys. Rev. D 74, 015005 (2006) arXiv:hep-ph/0602229.

[6] S. J. Brodsky, G. F. de Teramond, Phys. Rev. Lett. 96, 201601 (2006) hep-ph/0602252.

[7] S. J. Brodsky and G. F. de Teramond, Phys. Rev. D 77, 056007 (2008) arXiv:0707.3859 [hep-ph]].

[8] S. J. Brodsky, G. F. de Teramond and A. Deur, Phys. Rev. D 81, 096010 (2010) arXiv:1002.3948 [hep-ph]]; G. F. de Teramond, S. J. Brodsky, Nucl. Phys. Proc. Suppl. 199, 89 (2010). arXiv:0909.3900 [hep-ph]].

[9] G. F. de Teramond and S. J. Brodsky, AIP Conf. Proc. 1296, 128 (2010) arXiv:1006.2431 [hep-ph]].

[10] O. Andreev, Phys. Rev. D 73, 107901 (2006) arXiv:hep-th/0603170]; O. Andreev and V. I. Zakharov, Phys. Rev. D 74, 025023 (2006) arXiv:hep-ph/0604204.

[11] H. Forkel, M. Beyer and T. Frederico, JHEP 0707, 077 (2007) arXiv:0705.1857] [hep-ph]]; Int. J. Mod. Phys. E 16, 2794 (2007) arXiv:0705.4115 [hep-ph]]; W. de Paula, T. Frederico, H. Forkel and M. Beyer, Phys. Rev. D 79, 075019 (2009) arXiv:0806.3830 [hep-ph]].

[12] B. Galow, E. Megias, J. Nian and H. J. Pirner, Nucl. Phys. B 834, 330 (2010) arXiv:0911.0627 [hep-ph]]; J. Nian and H. J. Pirner, Nucl. Phys. A 833, 119 (2010) arXiv:0908.1330 [hep-ph]].

[13] H. R. Grigoryan and A. V. Radyushkin, Phys. Rev. D 76, 095007 (2007) arXiv:0706.1543 [hep-ph]].

[14] H. Forkel, Phys. Rev. D 78, 025001 (2008) arXiv:0711.1179 [hep-ph]]; H. J. Kwee and R. F. Lebed, Phys. Rev. D 77, 115007 (2008) arXiv:0712.1811 [hep-ph]]. P. Colangelo, F. De Fazio, F. Jugeau and S. Nicotri, Phys. Lett. B 652, 73 (2007) arXiv:hep-ph/0703316; T. Gherghetta, J. I. Kapusta and T. M. Kelley, Phys. Rev. D 79, 076003 (2009) arXiv:0902.1998 [hep-ph]]; Y. Q. Sui, Y. L. Wu, Z. F. Xie and Y. B. Yang, Phys. Rev. D 81, 014024 (2010) arXiv:0909.3887 [hepph]]; M. Fujita, K. Fukushima, T. Misumi and M. Murata, Phys. Rev. D 80, 035001 (2009) [arXiv:0903.2316 [hep-ph]]; S. S. Afonin, Int. J. Mod. Phys. A 25, 5683 (2010). arXiv:1001.3105 [hep-ph]]; C. Marquet, C. Roiesnel and S. Wallon, JHEP 1004, 051 (2010) arXiv:1002.0566 [hep-ph]]; H. R. Grigoryan, P. M. Hohler and M. A. Stephanov, Phys. Rev. D 82, 026005 (2010) arXiv:1003.1138 [hep-ph].

[15] A. Vega and I. Schmidt, Phys. Rev. D 78, 017703 (2008) arXiv:0806.2267 [hep-ph]]. 
[16] A. Vega and I. Schmidt, Phys. Rev. D 79, 055003 (2009) arXiv:0811.4638 [hep-ph]]; A. Vega, I. Schmidt, T. Branz, T. Gutsche and V. E. Lyubovitskij, Phys. Rev. D 80, 055014 (2009) arXiv:0906.1220 [hep-ph]]; A. Vega, I. Schmidt, Phys. Rev. D 82, 115023 (2010) arXiv:1005.3000 [hep-ph]].

[17] S. J. Brodsky and G. F. de Teramond, arXiv:0802.0514 [hep-ph]; G. F. de Teramond and S. J. Brodsky, AIP Conf. Proc. 1257, 59 (2010) arXiv:1001.5193 [hep-ph]].

[18] Z. Abidin and C. E. Carlson, Phys. Rev. D 79, 115003 (2009) arXiv:0903.4818 [hep-ph]].

[19] T. Branz, T. Gutsche, V. E. Lyubovitskij, I. Schmidt, A. Vega, Phys. Rev. D 82, 074022 (2010) [arXiv:1008.0268 [hep-ph]].

[20] A. Vega, I. Schmidt, T. Gutsche, V. E. Lyubovitskij, Phys. Rev. D 83, 036001 (2011) arXiv:1010.2815 [hep-ph]].

[21] F. Zuo, Phys. Rev. D 82, 086011 (2010). arXiv:0909.4240 [hep-ph]]; S. Nicotri, AIP Conf. Proc. 1317, 322 (2011) arXiv:1009.4829 [hep-ph]].

[22] A. Karch, E. Katz, D. T. Son, M. A. Stephanov, JHEP 1104, 066 (2011) arXiv:1012.4813 [hep-ph]].

[23] S. S. Afonin, Int. J. Mod. Phys. A 26, 3615 (2011) arXiv:1012.5065 [hep-ph]].

[24] S. J. Brodsky, G. F. de Teramond, Phys. Lett. B 582, 211 (2004) hep-th/0310227]; Phys. Rev. D 78, 025032 (2008) arXiv:0804.0452 [hep-ph]].

[25] L. Randall and R. Sundrum, Phys. Rev. Lett. 83, 3370 (1999) arXiv:hep-ph/9905221.

[26] M. Henningson and K. Sfetsos, Phys. Lett. B 431, 63 (1998) arXiv:hep-th/9803251; W. Mueck and K. S. Viswanathan, Phys. Rev. D 58, 106006 (1998) arXiv:hep-th/9805145; R. Contino and A. Pomarol, JHEP 0411, 058 (2004) arXiv:hep-th/0406257.

[27] I. L. Buchbinder, A. Pashnev and M. Tsulaia, Phys. Lett. B 523, 338 (2001) arXiv:hep-th/0109067]; R. R. Metsaev, Phys. Lett. B 590, 95 (2004) arXiv:hep-th/0312297]; C. Germani and A. Kehagias, Nucl. Phys. B 725, 15 (2005) arXiv:hep-th/0411269.

[28] F. E. Close, An Introduction To Quarks And Partons, (Academic Press, New York, 1979).

[29] J. F. Donoghue, E. Golowich and B. R. Holstein, Dynamics Of The Standard Model, Cambridge Monogr. Part. Phys. Nucl. Phys. Cosmol. 2, 1 (1992).

[30] T. Inoue, V. E. Lyubovitskij, T. Gutsche and A. Faessler, Int. J. Mod. Phys. E 15, 121 (2006) arXiv:hep-ph/0404051.

[31] M. Karliner, B. Keren-Zur, H. J. Lipkin and J. L. Rosner, Annals Phys. 324, 2 (2009) arXiv:0804.1575 [hep-ph]].

[32] A. Faessler, T. Gutsche, V. E. Lyubovitskij and K. Pumsa-ard, Phys. Rev. D 73, 114021 (2006) arXiv:hep-ph/0511319.

[33] K. Nakamura et al. (Particle Data Group), J. Phys. G 37, 075021 (2010).

[34] D. Ebert, R. N. Faustov, V. O. Galkin, A. P. Martynenko, Phys. Rev. D 70, 014018 (2004) hep-ph/0404280.

[35] D. Ebert, R. N. Faustov, V. O. Galkin, Phys. Rev. D 84, 014025 (2011) arXiv:1105.0583 [hep-ph]].

[36] A. P. Martynenko, Phys. Lett. B 663, 317-321 (2008) arXiv:0708.2033 [hep-ph]].

[37] M. A. Ivanov, V. E. Lyubovitskij, J. G. Körner and P. Kroll, Phys. Rev. D 56, 348 (1997) arXiv:hep-ph/9612463; M. A. Ivanov, J. G. Körner, V. E. Lyubovitskij and A. G. Rusetsky, Phys. Rev. D 57, 5632 (1998) arXiv:hep-ph/9709372; M. A. Ivanov, J. G. Körner, V. E. Lyubovitskij and A. G. Rusetsky, Phys. Rev. D 60, 094002 (1999) arXiv:hep-ph/9904421; A. Faessler, T. Gutsche, M. A. Ivanov, J. G. Körner, V. E. Lyubovitskij, D. Nicmorus and K. Pumsa-ard, Phys. Rev. D 73, 094013 (2006) arXiv:hep-ph/0602193; A. Faessler, T. Gutsche, M. A. Ivanov, J. G. Korner, V. E. Lyubovitskij, Phys. Rev. D 80, 034025 (2009) arXiv:0907.0563 [hep-ph]]; T. Branz, A. Faessler, T. Gutsche, M. A. Ivanov, J. G. Korner, V. E. Lyubovitskij, B. Oexl, Phys. Rev. D 81, 114036 (2010) arXiv:1005.1850 [hep-ph]].

[38] M. A. Ivanov, M. P. Locher and V. E. Lyubovitskij, Few Body Syst. 21, 131 (1996); A. Faessler, T. Gutsche, B. R. Holstein, V. E. Lyubovitskij, D. Nicmorus and K. Pumsa-ard, Phys. Rev. D 74, 074010 (2006) arXiv:hep-ph/0608015; A. Faessler, T. Gutsche, B. R. Holstein, M. A. Ivanov, J. G. Körner and V. E. Lyubovitskij, Phys. Rev. D 78, 094005 (2008) arXiv:0809.4159 [hep-ph]]. 
TABLE I: Mass spectrum of ground-state light baryon in $\mathrm{MeV}$

\begin{tabular}{|c|c|c|}
\hline Baryon & Our results & Data [33] \\
\hline$N$ & 939 & 939 \\
$\Lambda$ & 1114 & 1116 \\
$\Sigma$ & 1180 & 1189 \\
$\Xi$ & 1328 & 1322 \\
$\Delta$ & 1232 & 1232 \\
$\Sigma^{*}$ & 1381 & 1385 \\
$\Xi^{*}$ & 1533 & 1530 \\
$\Omega$ & 1688 & 1672 \\
\hline
\end{tabular}

TABLE II: Mass spectrum of $N$ and $\Delta$ families in $\mathrm{MeV}$

\begin{tabular}{|c|c|c|}
\hline Baryon & Our results & Data [33] \\
\hline$N_{1 / 2^{+}}(939)$ & 939 & 939 \\
$N_{1 / 2^{+}}(1440)$ & 1372 & $1440_{-20}^{+30}$ \\
$N_{1 / 2^{+}}(1710)$ & 1698 & $1710 \pm 30$ \\
$N_{1 / 2^{+}}(1880)$ & 1970 & \\
$N_{1 / 2^{+}}(2100)$ & 2209 & \\
$\Delta_{3 / 2^{+}}(1232)$ & 1232 & 1232 \\
$\Delta_{3 / 2^{+}}(1600)$ & 1587 & $1600_{-50}^{+100}$ \\
$\Delta_{3 / 2^{+}}(1920)$ & 1876 & $1920_{-20}^{+50}$ \\
\hline
\end{tabular}

TABLE III: Mass spectrum of $\Lambda, \Sigma$ and $\Sigma^{*}$ families in $\mathrm{MeV}$

\begin{tabular}{|c|c|c|}
\hline Baryon & Our results & Data [33] \\
\hline$\Lambda_{1 / 2^{+}}(1116)$ & 1114 & 1116 \\
$\Lambda_{1 / 2^{+}}(1600)$ & 1547 & $1600_{-40}^{+100}$ \\
$\Lambda_{1 / 2^{+}}(1810)$ & 1873 & $1810_{-60}^{+40}$ \\
$\Sigma_{1 / 2^{+}}(1189)$ & 1180 & 1189 \\
$\Sigma_{1 / 2^{+}}(1660)$ & 1593 & $1660 \pm 30$ \\
$\Sigma_{1 / 2^{+}}(1880)$ & 1910 & 1880 \\
$\Sigma_{3 / 2^{+}}(1385)$ & 1381 & 1385 \\
$\Sigma_{3 / 2^{+}}(1840)$ & 1741 & 1840 \\
$\Sigma_{3 / 2^{+}}(2080)$ & 2033 & 2080 \\
\hline
\end{tabular}


TABLE IV: Classification and mass spectrum (in $\mathrm{GeV}$ ) of ground-state single-heavy baryons

\begin{tabular}{|c|c|c|c|c|c|}
\hline Baryon & Content & $J^{P}$ & Ratio $\alpha_{B} / \alpha$ & Our results & Data [33], Ref. [35] \\
\hline$\Lambda_{c}$ & $c[u d]$ & $1 / 2^{+}$ & 1 & 2.286 & $2.286[33]$ \\
\hline$\Xi_{c}$ & $c[s q]$ & $1 / 2^{+}$ & $r_{s}$ & 2.511 & $2.468[33]$ \\
\hline$\Xi_{c}^{\prime}$ & $c\{s q\}$ & $1 / 2^{+}$ & $\frac{2 r_{c}+2 r_{c} r_{s}-r_{s}}{3}$ & 2.613 & $2.576[33]$ \\
\hline$\Sigma_{c}$ & $c\{q q\}$ & $1 / 2^{+}$ & $\frac{4 r_{c}-1}{3}$ & 2.446 & $2.454[33]$ \\
\hline$\Omega_{c}$ & $c\{s s\}$ & $1 / 2^{+}$ & $\frac{\left(4 r_{c}-r_{s}\right) r_{s}}{3}$ & 2.785 & $2.695[33]$ \\
\hline$\Lambda_{b}$ & $b[u d]$ & $1 / 2^{+}$ & 1 & 5.620 & $5.620[33]$ \\
\hline$\Xi_{b}$ & $b[s q]$ & $1 / 2^{+}$ & $r_{s}$ & 5.845 & $5.791[33]$ \\
\hline$\Xi_{b}^{\prime}$ & $b\{s q\}$ & $1 / 2^{+}$ & $\frac{2 r_{b}+2 r_{b} r_{s}-r_{s}}{3}$ & 5.972 & $5.936[35]$ \\
\hline$\Sigma_{b}$ & $b\{q q\}$ & $1 / 2^{+}$ & $\frac{4 r_{b}-1}{3}$ & 5.809 & $5.809[33]$ \\
\hline$\Omega_{b}$ & $b\{s s\}$ & $1 / 2^{+}$ & $\frac{\left(4 r_{b}-r_{s}\right) r_{s}}{3}$ & 6.139 & $6.071[33]$ \\
\hline$\Xi_{c}^{*}$ & $c\{s q\}$ & $3 / 2^{+}$ & $\frac{r_{c}+r_{s}+r_{c} r_{s}}{3}$ & 2.669 & $2.646[33]$ \\
\hline$\Sigma_{c}^{*}$ & $c\{q q\}$ & $3 / 2^{+}$ & $\frac{2 r_{c}+1}{3}$ & 2.511 & $2.518[33]$ \\
\hline$\Omega_{c}^{*}$ & $c\{s s\}$ & $3 / 2^{+}$ & $\frac{\left(2 r_{c}+r_{s}\right) r_{s}}{3}$ & 2.831 & $2.766[33]$ \\
\hline$\Xi_{b}^{*}$ & $b\{s q\}$ & $3 / 2^{+}$ & $\frac{r_{b}+r_{s}+r_{b} r_{s}}{3}$ & 5.991 & $5.963[35]$ \\
\hline$\Sigma_{b}^{*}$ & $b\{q q\}$ & $3 / 2^{+}$ & $\frac{2 r_{b}+1}{3}$ & 5.831 & $5.829[33]$ \\
\hline$\Omega_{b}^{*}$ & $b\{s s\}$ & $3 / 2^{+}$ & $\frac{\left(2 r_{b}+r_{s}\right) r_{s}}{3}$ & 6.155 & $6.088[35]$ \\
\hline
\end{tabular}


TABLE V: Classification and mass spectrum (in $\mathrm{GeV}$ ) of double-heavy and triple-heavy baryons

\begin{tabular}{|c|c|c|c|c|c|}
\hline Baryon & Content & $J^{P}$ & Ratio $\alpha_{B} / \alpha$ & Our results & Data [33], Refs. [34, 36] \\
\hline$\Xi_{c c}$ & $q\{c c\}$ & $1 / 2^{+}$ & $\frac{\left(4-r_{c}\right) r_{c}}{3}$ & 3.747 & $3.5189[33]$ \\
\hline$\Xi_{b c}$ & $q\{b c\}$ & $1 / 2^{+}$ & $\frac{2 r_{b}+2 r_{c}-r_{b} r_{c}}{3}$ & 7.094 & $6.933[34]$ \\
\hline$\Xi_{b c}^{\prime}$ & $q[b c]$ & $1 / 2^{+}$ & $r_{b} r_{c}$ & 7.121 & $6.963[34]$ \\
\hline$\Xi_{b b}$ & $q\{b b\}$ & $1 / 2^{+}$ & $\frac{\left(4-r_{b}\right) r_{b}}{3}$ & 10.442 & $10.202[\underline{34}]$ \\
\hline$\Xi_{c c}^{*}$ & $q\{c c\}$ & $3 / 2^{+}$ & $\frac{\left(2+r_{c}\right) r_{c}}{3}$ & 3.814 & $3.727[34]$ \\
\hline$\Xi_{b c}^{*}$ & $q\{b c\}$ & $3 / 2^{+}$ & $\frac{r_{b}+r_{c}+r_{b} r_{c}}{3}$ & 7.139 & $6.980[34]$ \\
\hline$\Xi_{b b}^{*}$ & $q\{b b\}$ & $3 / 2^{+}$ & $\frac{\left(2+r_{b}\right) r_{b}}{3}$ & 10.465 & $10.237[34]$ \\
\hline$\Omega_{c c}$ & $s\{c c\}$ & $1 / 2^{+}$ & $\frac{\left(4 r_{s}-r_{c}\right) r_{c}}{3}$ & 3.936 & $3.778[34]$ \\
\hline$\Omega_{b c}$ & $s\{b c\}$ & $1 / 2^{+}$ & $\frac{2\left(r_{b}+r_{c}\right) r_{s}-r_{b} r_{c}}{3}$ & 7.257 & 7.088 [34] \\
\hline$\Omega_{b c}^{\prime}$ & $s[b c]$ & $1 / 2^{+}$ & $r_{b} r_{c}$ & 7.296 & $7.116[34]$ \\
\hline$\Omega_{b b}$ & $s\{b b\}$ & $1 / 2^{+}$ & $\frac{\left(4 r_{s}-r_{b}\right) r_{b}}{3}$ & 10.622 & $10.359[34]$ \\
\hline$\Omega_{c c}^{*}$ & $s\{c c\}$ & $3 / 2^{+}$ & $\frac{\left(2 r_{s}+r_{c}\right) r_{c}}{3}$ & 3.982 & $3.872[34]$ \\
\hline$\Omega_{b c}^{*}$ & $s\{b c\}$ & $3 / 2^{+}$ & $\frac{r_{b} r_{s}+r_{c} r_{s}+r_{b} r_{c}}{3}$ & 7.310 & $7.130[34]$ \\
\hline$\Omega_{b b}^{*}$ & $s\{b b\}$ & $3 / 2^{+}$ & $\frac{\left(2 r_{s}+r_{b}\right) r_{b}}{3}$ & 10.638 & $10.389[34]$ \\
\hline$\Omega_{c c b}$ & $b\{c c\}$ & $1 / 2^{+}$ & $\frac{\left(4 r_{b}-r_{c}\right) r_{c}}{3}$ & 8.469 & $8.018[36]$ \\
\hline$\Omega_{c b b}$ & $c\{b b\}$ & $1 / 2^{+}$ & $\frac{\left(4 r_{c}-r_{b}\right) r_{b}}{3}$ & 11.801 & $11.280[36]$ \\
\hline$\Omega_{c c c}^{*}$ & $c c c$ & $3 / 2^{+}$ & $r_{c}^{2}$ & 5.144 & $4.803[36]$ \\
\hline$\Omega_{c c b}^{*}$ & $b\{c c\}$ & $3 / 2^{+}$ & $\frac{\left(2 r_{b}+r_{c}\right) r_{c}}{3}$ & 8.475 & $8.025[36]$ \\
\hline$\Omega_{c b b}^{*}$ & $c\{b b\}$ & $3 / 2^{+}$ & $\frac{\left(2 r_{c}+r_{b}\right) r_{b}}{3}$ & 11.806 & $11.287[36]$ \\
\hline$\Omega_{b b b}^{*}$ & $b b b$ & $3 / 2^{+}$ & $r_{b}^{2}$ & 15.139 & $14.569[36]$ \\
\hline
\end{tabular}


TABLE VI: Mass spectrum (in GeV) of $\Lambda_{Q}, \Sigma_{Q}, \Xi_{Q}, \Xi_{Q}^{\prime}$ and $\Omega_{Q}$ heavy baryon families with $J^{P}=\frac{1}{2}^{+}$

\begin{tabular}{|c|c|c||c|c|c|}
\hline Baryon $(n L)$ & Our results & Ref. [35] & Baryon $(n L)$ & Our results & Ref. [35] \\
\hline$\Lambda_{c}(1 S)$ & 2.286 & 2.286 & $\Lambda_{b}(1 S)$ & 5.620 & 5.620 \\
$\Lambda_{c}(2 S)$ & 2.719 & 2.769 & $\Lambda_{b}(2 S)$ & 6.053 & 6.089 \\
$\Lambda_{c}(3 S)$ & 3.045 & 3.130 & $\Lambda_{b}(3 S)$ & 6.379 & 6.455 \\
$\Lambda_{c}(4 S)$ & 3.317 & 3.437 & $\Lambda_{b}(4 S)$ & 6.651 & 6.756 \\
$\Sigma_{c}(1 S)$ & 2.446 & 2.443 & $\Sigma_{b}(1 S)$ & 5.809 & 5.808 \\
$\Sigma_{c}(2 S)$ & 2.833 & 2.901 & $\Sigma_{b}(2 S)$ & 6.188 & 6.213 \\
$\Sigma_{c}(3 S)$ & 3.138 & 3.271 & $\Sigma_{b}(3 S)$ & 6.490 & 6.575 \\
$\Sigma_{c}(4 S)$ & 3.400 & 3.581 & $\Sigma_{b}(4 S)$ & 6.748 & 6.869 \\
$\Xi_{c}(1 S)$ & 2.584 & 2.476 & $\Xi_{b}(1 S)$ & 5.940 & 5.803 \\
$\Xi_{c}(2 S)$ & 2.980 & 2.959 & $\Xi_{b}(2 S)$ & 6.331 & 6.266 \\
$\Xi_{c}(3 S)$ & 3.290 & 3.323 & $\Xi_{b}(3 S)$ & 6.638 & 6.601 \\
$\Xi_{c}(4 S)$ & 3.553 & 3.632 & $\Xi_{b}(4 S)$ & 6.900 & 6.913 \\
$\Xi_{c}^{\prime}(1 S)$ & 2.613 & 2.579 & $\Xi_{b}^{\prime}(1 S)$ & 5.972 & 5.936 \\
$\Xi_{c}^{\prime}(2 S)$ & 3.002 & 2.983 & $\Xi_{b}^{\prime}(2 S)$ & 6.354 & 6.329 \\
$\Xi_{c}^{\prime}(3 S)$ & 3.308 & 3.377 & $\Xi_{b}^{\prime}(3 S)$ & 6.657 & 6.687 \\
$\Xi_{c}^{\prime}(4 S)$ & 3.569 & 3.695 & $\Xi_{b}^{\prime}(4 S)$ & 6.916 & 6.978 \\
$\Omega_{c}(1 S)$ & 2.785 & 2.698 & $\Omega_{b}(1 S)$ & 6.139 & 6.064 \\
$\Omega_{c}(2 S)$ & 3.175 & 3.088 & $\Omega_{b}(2 S)$ & 6.524 & 6.450 \\
$\Omega_{c}(3 S)$ & 3.481 & 3.489 & $\Omega_{b}(3 S)$ & 6.828 & 6.804 \\
$\Omega_{c}(4 S)$ & 3.742 & 3.814 & $\Omega_{b}(4 S)$ & 7.087 & 7.091 \\
\hline
\end{tabular}

TABLE VII: Mass spectrum (in GeV) of $\Sigma_{Q}^{*}, \Xi_{Q}^{*}$, and $\Omega_{Q}^{*}$ heavy baryon families with $J^{P}=\frac{3}{2}^{+}$

\begin{tabular}{|c|c|c||c|c|c|}
\hline Baryon $(n L)$ & Our results & Ref. [35] & Baryon $(n L)$ & Our results & Ref. [35] \\
\hline$\Sigma_{c}^{*}(1 S)$ & 2.511 & 2.519 & $\Sigma_{b}^{*}(1 S)$ & 5.831 & 5.834 \\
$\Sigma_{c}^{*}(2 S)$ & 2.881 & 2.936 & $\Sigma_{b}^{*}(2 S)$ & 6.205 & 6.226 \\
$\Sigma_{c}^{*}(3 S)$ & 3.178 & 3.293 & $\Sigma_{b}^{*}(3 S)$ & 6.504 & 6.583 \\
$\Sigma_{c}^{*}(4 S)$ & 3.434 & 3.598 & $\Sigma_{b}^{*}(4 S)$ & 6.760 & 6.876 \\
$\Xi_{c}^{*}(1 S)$ & 2.669 & 2.649 & $\Xi_{b}^{*}(1 S)$ & 5.991 & 5.963 \\
$\Xi_{c}^{*}(2 S)$ & 3.043 & 3.026 & $\Xi_{b}^{*}(2 S)$ & 6.369 & 6.342 \\
$\Xi_{c}^{*}(3 S)$ & 3.343 & 3.396 & $\Xi_{b}^{*}(3 S)$ & 6.669 & 6.695 \\
$\Xi_{c}^{*}(4 S)$ & 3.600 & 3.709 & $\Xi_{b}^{*}(4 S)$ & 6.927 & 6.984 \\
$\Omega_{c}^{*}(1 S)$ & 2.831 & 2.768 & $\Omega_{b}^{*}(1 S)$ & 6.155 & 6.088 \\
$\Omega_{c}^{*}(2 S)$ & 3.209 & 3.123 & $\Omega_{b}^{*}(2 S)$ & 6.535 & 6.461 \\
$\Omega_{c}^{*}(3 S)$ & 3.509 & 3.510 & $\Omega_{b}^{*}(3 S)$ & 6.837 & 6.811 \\
$\Omega_{c}^{*}(4 S)$ & 3.767 & 3.830 & $\Omega_{b}^{*}(4 S)$ & 7.096 & 7.096 \\
\hline
\end{tabular}

